[Supporting Information]

\title{
Stereoselective Synthesis of Premisakinolide A, the Monomeric Counterpart of the Marine 40-Membered Dimeric Macrolide Misakinolide
}

\author{
Ryoichi Nakamura, Keiji Tanino, and Masaaki Miyashita* \\ Division of Chemistry, Graduate School of Science, Hokkaido University, Sapporo 060-0810, Japan
}

\section{Experimental}

General. All reactions were carried out under a dry argon atmosphere. Flash chromatography was performed using 40-100 $\mu \mathrm{m}$ mesh KANTO silica gel $60 \mathrm{~N}$ or 0.063-0.200 $\mu \mathrm{m}$ mesh Merck silica gel 60 for purification of products.

Materials. Diethyl ether and tetrahydrofuran were distilled from sodium and benzophenone immediately before use. DMF, toluene, benzene, and triethylamine were distilled from $\mathrm{CaH}_{2}$. Dehydrated $\mathrm{CH}_{2} \mathrm{Cl}_{2}$ was purchased from Kanto Chemical Co. Ltd.

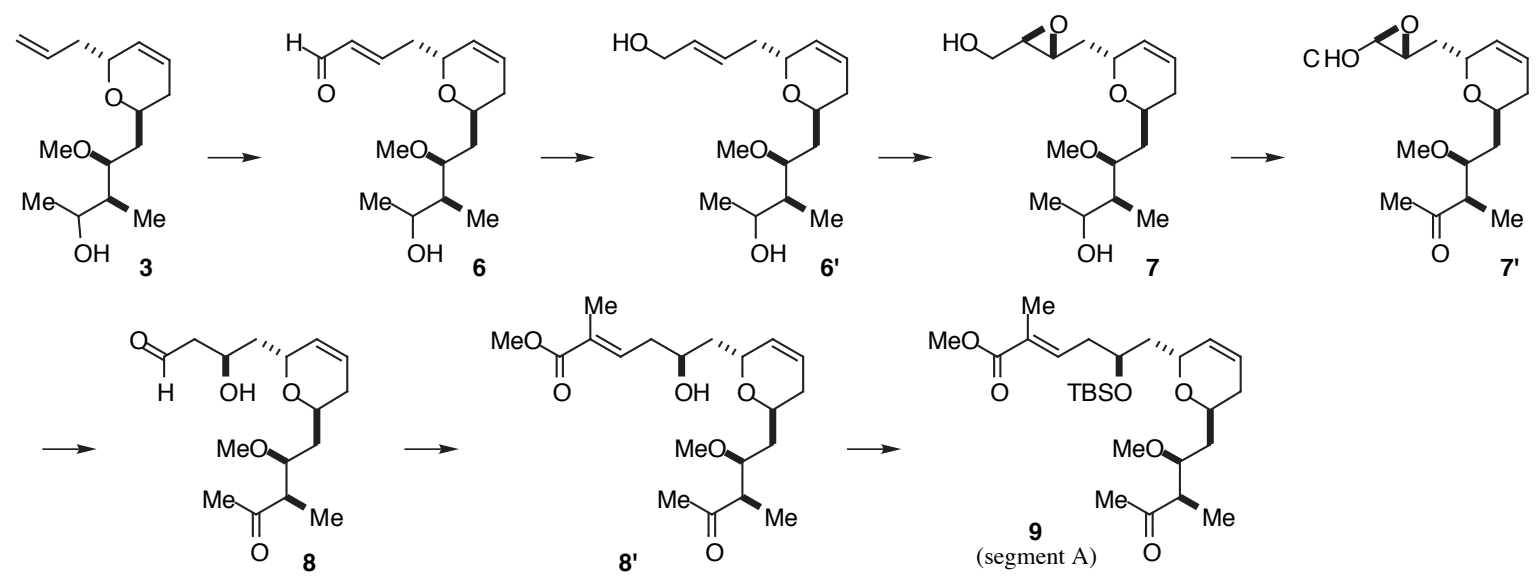

\section{Unsaturated aldehyde 6}

To a stirred solution of compound $\mathbf{3}^{1}$ (2.94 g, $\left.11.6 \mathrm{mmol}\right)$ and 2nd Generation Grubbs' Catalyst (196 mg, 0.23 mmol, $2 \mathrm{~mol} \%)$ in $\mathrm{CH}_{2} \mathrm{Cl}_{2}(100 \mathrm{~mL})$ was added crotonaldehyde $(1.92 \mathrm{~mL}, 23.1 \mathrm{mmol})$ at room temperature. After refuxing for $2 \mathrm{~h}$, another portion of crotonaldehyde $(0.96 \mathrm{~mL}, 11.6 \mathrm{mmol})$ was added and the resulting mixture was refluxed for additional $30 \mathrm{~min}$. The reaction mixture was then concentrated and purified directly by silica gel column chromatography (EtOAc/hexane 20\%, 30\%, 40\%, then 50\%) to give the unsaturated aldehyde $6(2.54 \mathrm{~g}, 78 \%)$ as an oil: IR (neat) 3490 (br), 2972, 2825, 1689, 1134, 1089, $977 \mathrm{~cm}^{-1} ;{ }^{1} \mathrm{H}$ NMR $\delta(400 \mathrm{MHz}$, 
$\left.\mathrm{CDCl}_{3}\right) 9.53(1 \mathrm{H}, \mathrm{d}, J=8.0 \mathrm{~Hz}), 6.86(1 \mathrm{H}, \mathrm{dt}, J=15.6,7.2 \mathrm{~Hz}), 6.19(1 \mathrm{H}, \mathrm{ddt}, J=15.6,8.0,1.2 \mathrm{~Hz}), 5.91(1$ H, dddt, $J=10.4,2.8,2.8,2.4 \mathrm{~Hz}), 5.70$ (1 H, dddd, $J=10.4,2.8,2.4,2.0 \mathrm{~Hz}), 4.39$ (1 H, br s), 3.98 (1 H, qd, $J$ = 6.4, $2.0 \mathrm{~Hz}), 3.71-3.64(1 \mathrm{H}, \mathrm{m}), 3.60(1 \mathrm{H}, \mathrm{ddd}, J=9.6,4.0,2.8 \mathrm{~Hz}), 3.36(3 \mathrm{H}, \mathrm{s}), 3.16(1 \mathrm{H}, \mathrm{s}), 2.65(1 \mathrm{H}$, dddd, $J=15.6,8.8,7.2,1.6 \mathrm{~Hz}), 2.52(1 \mathrm{H}$, dddd, $J=15.6,7.2,4.8,1.6 \mathrm{~Hz}), 2.09-2.00(2 \mathrm{H}, \mathrm{m}), 1.95(1 \mathrm{H}$, ddd, $J=14.4,10.0,4.0 \mathrm{~Hz}), 1.61(1 \mathrm{H}, \mathrm{ddd}, J=14.4,9.6,3.2 \mathrm{~Hz}), 1.54(1 \mathrm{H}, \mathrm{qdd}, J=7.2,2.8,2.0 \mathrm{~Hz}), 1.15(3 \mathrm{H}, \mathrm{d}$, $J=6.4 \mathrm{~Hz}), 0.91(3 \mathrm{H}, \mathrm{d}, J=7.2 \mathrm{~Hz}) ;{ }^{13} \mathrm{C} \mathrm{NMR} \delta\left(67.8 \mathrm{MHz}, \mathrm{CDCl}_{3}\right) 193.3,154.3,134.4,127.8,125.2,82.4$, 71.2, 71.0, 64.7, 56.3, 40.2, 37.1, 35.1, 30.8, 20.9, 5.6; HRMS $\left(\mathrm{FD}^{+}\right)[\mathrm{M}+\mathrm{H}]^{+}$found 283.1893, $\mathrm{C}_{16} \mathrm{H}_{27} \mathrm{O}_{4}$ requires 283.1909 .

\section{Allyl alcohol 6'}

To a solution of unsaturated aldehyde $6(2.54 \mathrm{~g}, 9.0 \mathrm{mmol})$ in dry THF $(45 \mathrm{~mL})$ was added DIBAL $(0.94 \mathrm{M}$ solution in hexane, $23 \mathrm{~mL}, 21.6 \mathrm{mmol}$ ) at $0{ }^{\circ} \mathrm{C}$. After stirring for $20 \mathrm{~min}$, the reaction mixture was quenched with satd. aq. potassium sodium tartrate solution, and then the mixture was separated. The organic layer was washed with brine and the aqueous layers were extracted with AcOEt (x4). The combined organic layers were dried $\left(\mathrm{MgSO}_{4}\right)$ and concentrated in vacuo. The crude oil was purified by neutral silica gel column chromatography (EtOAc/hexane 50\%, 70\%, then 80\%) to give allyl alcohol 6' (2.34 g, 92\%) as an oil: IR (neat) 3400 (br), 2972, 2368, 1090, $970 \mathrm{~cm}^{-1} ;{ }^{1} \mathrm{H}$ NMR $\delta$ (400 MHz, $\mathrm{CDCl}_{3}$ ) 5.87-5.81 (1 H, m), 5.77-5.67 (3 H, m), 4.27-4.20 (1 H, m), 4.16-4.08 (2 H, m), $4.02(1 \mathrm{H}, \mathrm{qd}, J=6.4,2.0 \mathrm{~Hz}), 3.71-3.60(2 \mathrm{H}, \mathrm{m}), 3.36(3 \mathrm{H}, \mathrm{s}), 3.38$ $3.30(1 \mathrm{H}, \mathrm{m}), 2.40-2.32(1 \mathrm{H}, \mathrm{m}), 2.28-2.21(1 \mathrm{H}, \mathrm{m}), 2.02-1.96(2 \mathrm{H}, \mathrm{m}), 1.94(1 \mathrm{H}, \mathrm{ddd}, J=14.0,10.0,4.0$ $\mathrm{Hz}), 1.80-1.69(1 \mathrm{H}, \mathrm{m}), 1.65-1.55(2 \mathrm{H}, \mathrm{m}), 1.17(3 \mathrm{H}, \mathrm{d}, J=6.4 \mathrm{~Hz}), 0.91(3 \mathrm{H}, \mathrm{d}, J=7.2 \mathrm{~Hz}) ;{ }^{13} \mathrm{C} \mathrm{NMR} \delta$ $\left(67.8 \mathrm{MHz}, \mathrm{CDCl}_{3}\right)$ 131.6, 128.9, 128.5, 124.3, 82.3, 72.4, 71.6, 64.5, 63.3, 56.4, 40.2, 36.9, 35.3, 31.2, 20.8, 5.9; HRMS $\left(\mathrm{FD}^{+}\right)[\mathrm{M}+\mathrm{H}]^{+}$found 285.2050, $\mathrm{C}_{16} \mathrm{H}_{29} \mathrm{O}_{4}$ requires 285.2066.

\section{Epoxy alcohol 7}

To a suspension of powdered molecular sieves $4 \mathrm{~A}(2.38 \mathrm{~g})$ and $\mathrm{CH}_{2} \mathrm{Cl}_{2}(20 \mathrm{~mL})$ was added titanium(IV) isopropoxide $(2.72 \mathrm{~mL}, 9.87 \mathrm{mmol})$ and (D)-(-)-diethyl tartrate $(1.7 \mathrm{~mL}, 9.87 \mathrm{mmol})$ at $-25{ }^{\circ} \mathrm{C}$ and the mixture was stirred for $10 \mathrm{~min}$. Then a solution of 6' $(2.34 \mathrm{~g}, 8.23 \mathrm{mmol})$ in $\mathrm{CH}_{2} \mathrm{Cl}_{2}(20 \mathrm{~mL})$ was added and the mixture was further stirred for $20 \mathrm{~min}$ at the same temperature, to which tert-butyl hydroperoxide (2.20 M solution in toluene, $9.0 \mathrm{~mL}, 19.7 \mathrm{mmol}$ ) was added and the resulting mixture was stirred at $-25{ }^{\circ} \mathrm{C}$ for an additional $2 \mathrm{~h}$. The reaction mixture was treated with aqueous tartaric acid $(10 \%)$ at $-25{ }^{\circ} \mathrm{C}$ and warmed up to room temperature and stirred for an additional $30 \mathrm{~min}$. The mixture was separated and the aqueous layer was extracted with $\mathrm{CH}_{2} \mathrm{Cl}_{2}$ (x8). The combined organic layers were concentrated in vacuo. The residue was dissolved in ether $(80 \mathrm{~mL})$ and treated with $1 \mathrm{~N} \mathrm{NaOH}(30 \mathrm{~mL})$ at $0{ }^{\circ} \mathrm{C}$. After being stirred vigorously for 30 min, the mixture was separated and the organic layer was washed with brine. The aqueous washes were 
extracted with $\mathrm{CH}_{2} \mathrm{Cl}_{2}(\mathrm{x} 5)$ and the combined organic layers were dried $\left(\mathrm{MgSO}_{4}\right)$ and concentrated in vacuo. The crude product was purified by silica gel column chromatography (EtOAc/hexane 80\%, then 100\%) to give epoxy alcohol 7 (2.43 g, 98\%) as an oil: IR (neat) 3400 (br), 2974, 2929, 2827, 1460, 1184, 1090, $756 \mathrm{~cm}^{-1} ;{ }^{1} \mathrm{H}$ NMR $\delta\left(400 \mathrm{MHz}, \mathrm{CDCl}_{3}\right) 5.86$ (1 H, dddt, $\left.J=10.4,2.8,2.4,2.0 \mathrm{~Hz}\right), 5.69$ (1 H, dm, $\left.J=10.4 \mathrm{~Hz}\right), 4.46-4.39$ (1 H, m), $4.01(1 \mathrm{H}, \mathrm{qd}, J=6.4,2.0 \mathrm{~Hz}), 3.88(1 \mathrm{H}, \mathrm{ddd}, J=12.0,4.0,2.8 \mathrm{~Hz}), 3.72-3.61(3 \mathrm{H}, \mathrm{m}), 3.37(3 \mathrm{H}, \mathrm{s})$, $3.14(1 \mathrm{H}, \mathrm{s}), 3.12(1 \mathrm{H}, \mathrm{ddd}, J=4.0,3.2,2.4 \mathrm{~Hz}), 2.96(1 \mathrm{H}, \mathrm{ddd}, J=4.0,2.8,2.8 \mathrm{~Hz}), 2.05-1.94(3 \mathrm{H}, \mathrm{m})$, 1.89-1.82 (1 H, m), 1.64-1.56 (3 H, m), 1.49 (1 H, ddd, $J=14.4,8.0,4.0 \mathrm{~Hz}), 1.17$ (3 H, d, $J=6.4 \mathrm{~Hz}), 0.92(3$ $\mathrm{H}, \mathrm{d}, J=7.2 \mathrm{~Hz}) ;{ }^{13} \mathrm{C} \mathrm{NMR} \delta\left(67.8 \mathrm{MHz}, \mathrm{CDCl}_{3}\right)$ 128.8, 124.4, 77.3, 71.3, 70.2, 64.7, 61.6, 58.7, 56.4, 53.3, 40.5, 36.3, 35.4, 30.9, 20.8, 6.3; HRMS $\left(\mathrm{FD}^{+}\right)[\mathrm{M}]^{+}$found 300.1957, $\mathrm{C}_{16} \mathrm{H}_{28} \mathrm{O}_{5}$ requires 300.1937 .

\section{Epoxy aldehyde 7'}

To a solution of 7 (506.1 mg, $1.68 \mathrm{mmol})$ in dry $\mathrm{CH}_{2} \mathrm{Cl}_{2}(10 \mathrm{~mL})$ was added Dess-Martin periodinane $(2.57 \mathrm{~g}$, $6.07 \mathrm{mmol}$ ). The mixture was stirred at room temperature for $1 \mathrm{~h}$ and treated with a 1:1 mixture of satd. $\mathrm{NaHCO}_{3}$ and satd. $\mathrm{Na}_{2} \mathrm{~S}_{2} \mathrm{O}_{3}$. The organic layer was separated and the aqueous layer was extracted with AcOEt. The combined organic layers were dried $\left(\mathrm{MgSO}_{4}\right)$ and concentrated in vacuo to give epoxy aldehyde 7' which was used for the next step without purification: ${ }^{1} \mathrm{H}$ NMR $\delta\left(270 \mathrm{MHz}, \mathrm{CDCl}_{3}\right) 9.04(1 \mathrm{H}, \mathrm{d}, J=6.3 \mathrm{~Hz}), 5.88$ $(1 \mathrm{H}, \mathrm{ddt}, J=10.2,5.1,2.5 \mathrm{~Hz}), 5.67(1 \mathrm{H}, \mathrm{ddt}, J=10.1,2.5,1.6 \mathrm{~Hz}), 4.45(1 \mathrm{H}, \mathrm{br} \mathrm{d}, J=9.4 \mathrm{~Hz}), 3.82-3.70(1 \mathrm{H}$, m), $3.64(1 \mathrm{H}, \mathrm{ddd}, J=5.8,5.8,5.1 \mathrm{~Hz}), 3.50(1 \mathrm{H}, \mathrm{ddd}, J=7.7,3.8,2.0 \mathrm{~Hz}), 3.34(3 \mathrm{H}, \mathrm{s}), 3.20(1 \mathrm{H}, \mathrm{dd}, J=6.3$, $2.0 \mathrm{~Hz}), 2.75(1 \mathrm{H}, \mathrm{qd}, J=7.3,5.3 \mathrm{~Hz}), 2.21-2.08(1 \mathrm{H}, \mathrm{m}), 2.20(3 \mathrm{H}, \mathrm{s}), 2.08-1.91(2 \mathrm{H}, \mathrm{m}), 1.83(1 \mathrm{H}, \mathrm{ddd}, J=$ 14.2, 7.4, $6.8 \mathrm{~Hz}), 1.65-1.52(2 \mathrm{H}, \mathrm{m}), 1.12(3 \mathrm{H}, \mathrm{d}, J=7.1 \mathrm{~Hz}) ;{ }^{13} \mathrm{C} \mathrm{NMR} \delta\left(67.8 \mathrm{MHz}, \mathrm{CDCl}_{3}\right) 211.3,198.1$, $128.2,124.8,78.8,69.8,65.0,59.2,57.2,54.2,49.5,36.5,35.8,30.2,29.5,11.3$.

\section{Unsaturated ester 8'}

A solution of $\mathrm{Na}\left[\mathrm{PhSeB}(\mathrm{OEt})_{3}\right](0.4 M$ solution in $\mathrm{EtOH}, 10.1 \mathrm{~mL}, 4.04 \mathrm{mmol})$, which was prepared from $(\mathrm{PhSe})_{2}(780.3 \mathrm{mg}, 2.5 \mathrm{mmol})$ in $\mathrm{EtOH}(12.5 \mathrm{~mL}), \mathrm{NaBH}_{4}(189.2 \mathrm{mg}, 5 \mathrm{mmol})$ and AcOH $(429.3 \mu \mathrm{L}, 7.5$ mmol) by stirring for $5 \mathrm{~min}$ at $0{ }^{\circ} \mathrm{C}$, was added to a solution of crude 7 ' in $\mathrm{EtOH}(7 \mathrm{~mL})$ at $0{ }^{\circ} \mathrm{C}$. After stirring for $1 \mathrm{~h}$, the reaction mixture was bubbled with $\mathrm{O}_{2}$, diluted with AcOEt and washed with brine, then the layers were separated. The aqueous layers were extracted with AcOEt (x3). The combined organic layers were dried $\left(\mathrm{MgSO}_{4}\right)$ and concentrated in vacuo to give hydroxy aldehyde 8 , which was used for the next step without purification.

To a solution of the crude aldehyde 8 in dry toluene $(10 \mathrm{~mL})$ was added $\mathrm{Ph}_{3} \mathrm{P}=\mathrm{C}(\mathrm{Me}) \mathrm{CO}_{2} \mathrm{Me}(1.9 \mathrm{~g}, 5.46$ $\mathrm{mmol}$ ). After being stirred for $1.5 \mathrm{~h}$ at room temperature, the mixture was filtered through a pad of Celite by the aid of AcOEt and the filtrate was concentrated in vacuo. The residue was purified by neutral silica gel column chromatography (EtOAc/hexane 25\%, 30\%, 35\%, 40\%, 45\%) to give unsaturated ester $\mathbf{8}$ (427 $\mathrm{mg}, 69 \%$ ) as a 
colourless oil: $[\alpha]_{\mathrm{D}}{ }^{30}=-64.9^{\circ}\left(\mathrm{c} 1.1, \mathrm{CHCl}_{3}\right)$; IR (neat) 3500 (br), 2945, 1717, 1437, 1194, 1088, $752 \mathrm{~cm}^{-1} ;{ }^{1} \mathrm{H}$ NMR $\delta\left(270 \mathrm{MHz}, \mathrm{CDCl}_{3}\right) 6.76(1 \mathrm{H}, \mathrm{t}, J=6.8 \mathrm{~Hz}), 5.81-5.70(1 \mathrm{H}, \mathrm{m}), 5.56(1 \mathrm{H}, \mathrm{d}, J=10.1 \mathrm{~Hz}), 4.43(1 \mathrm{H}$, br d, $J=8.4 \mathrm{~Hz}), 4.05-3.91(1 \mathrm{H}, \mathrm{m}), 3.83-3.71(1 \mathrm{H}, \mathrm{m}), 3.66(3 \mathrm{H}, \mathrm{s}), 3.57(1 \mathrm{H}, \mathrm{ddd}, J=6.3,5.9,5.6 \mathrm{~Hz}), 3.27(3 \mathrm{H}$, s), 2.70-2.54 (1H, m), 2.69 (1H, ddd, $J=14.0,7.1,4.8 \mathrm{~Hz}), 2.44-2.22(2 \mathrm{H}, \mathrm{m}), 2.13(3 \mathrm{H}, \mathrm{s}), 2.12-2.03(1 \mathrm{H}, \mathrm{m})$, 1.91-1.65 (3H, m), $1.79(3 \mathrm{H}, \mathrm{s}), 1.58-1.42(2 \mathrm{H}, \mathrm{m}), 1.04(3 \mathrm{H}, \mathrm{d}, J=7.1 \mathrm{~Hz}) ;{ }^{13} \mathrm{C} \mathrm{NMR} \delta\left(67.8 \mathrm{MHz}, \mathrm{CDCl}_{3}\right)$ $211.0,168.3,138.1,129.3,128.5,123.7,79.1,68.6,67.6,65.7,57.2,51.6,49.4,39.9,36.8,35.7,30.0,29.5$, 12.6, 11.2.

\section{Segment A (9)}

To a solution of alcohol 8' (469 mg, $1.27 \mathrm{mmol})$ in dry $\mathrm{CH}_{2} \mathrm{Cl}_{2}(6 \mathrm{~mL})$ was added 2,6-lutidine (0.44 mL, 3.82 mmol) and TBSOTf $(0.38 \mathrm{~mL}, 1.65 \mathrm{mmol})$ at $-78{ }^{\circ} \mathrm{C}$. After being stirred for $10 \mathrm{~min}$ at $-78{ }^{\circ} \mathrm{C}$, the reaction was quenched with $\mathrm{H}_{2} \mathrm{O}$. The mixture was separated and the aqueous layer was extracted with ether (x6). The combined organic layers were dried $\left(\mathrm{MgSO}_{4}\right)$ and concentrated in vacuo to leave an oil which was purified by silica gel column chromatography (EtOAc/hexane 15\%) to give segment $\mathrm{A}(\mathbf{9})(541.5 \mathrm{mg}, 88 \%)$ as an oil: $[\alpha]_{\mathrm{D}}{ }^{30}$ $=-62.6^{\circ}$ (c 1.4, $\mathrm{CHCl}_{3}$ ); IR (neat) 3450 (br), 2936, 1717, 1256, 1088, 837, $777 \mathrm{~cm}^{-1} ;{ }^{1} \mathrm{H}$ NMR $\delta(270 \mathrm{MHz}$, $\left.\mathrm{CDCl}_{3}\right) 6.78(1 \mathrm{H}, \mathrm{tq}, J=7.5,1.3 \mathrm{~Hz}), 5.79(1 \mathrm{H}, \mathrm{ddt}, J=10.1,4.9,2.5 \mathrm{~Hz}), 5.65(1 \mathrm{H}, \mathrm{br} \mathrm{d}, J=10.4 \mathrm{~Hz}), 4.35$ $(1 \mathrm{H}$, br d, $J=10.6 \mathrm{~Hz}), 4.09(1 \mathrm{H}, \mathrm{dtd}, J=9.3,5.6,2.5 \mathrm{~Hz}), 3.76(1 \mathrm{H}, \mathrm{ddd}, J=7.3,5.8,4.3 \mathrm{~Hz}), 3.72(3 \mathrm{H}, \mathrm{s})$, $3.58(1 \mathrm{H}, \mathrm{ddt}, J=8.4,8.4,4.3 \mathrm{~Hz}), 3.31(3 \mathrm{H}, \mathrm{s}), 2.66(1 \mathrm{H}, \mathrm{qd}, J=7.0,4.1 \mathrm{~Hz}), 2.38(2 \mathrm{H}, \mathrm{t}, J=6.4 \mathrm{~Hz}), 2.20$ $(3 \mathrm{H}, \mathrm{s}), 2.08-1.89(2 \mathrm{H}, \mathrm{m}), 1.83(3 \mathrm{H}, \mathrm{d}, J=1.2 \mathrm{~Hz}), 1.75(1 \mathrm{H}, \mathrm{ddd}, J=13.5,8.2,6.1 \mathrm{~Hz}), 1.69-1.52(2 \mathrm{H}, \mathrm{m})$, $1.41(1 \mathrm{H}, \mathrm{ddd}, J=14.4,9.7,2.8 \mathrm{~Hz}), 1.11(3 \mathrm{H}, \mathrm{d}, J=7.1 \mathrm{~Hz}), 0.90(9 \mathrm{H}, \mathrm{s}), 0.13(3 \mathrm{H}, \mathrm{s}), 0.12(3 \mathrm{H}, \mathrm{s}) ;{ }^{13} \mathrm{C}$ NMR $\delta\left(67.8 \mathrm{MHz}_{\mathrm{CDCl}}\right)$ 211.0, 168.4, 138.2, 130.2, 129.0, 123.4, 78.5, 69.1, 67.7, 63.9, 57.1, 51.7, 49.5, 40.6, $37.6,36.6,30.9,29.2,25.8(3 \mathrm{C}), 18.0,12.7,10.7,-4.4,-4.7$.

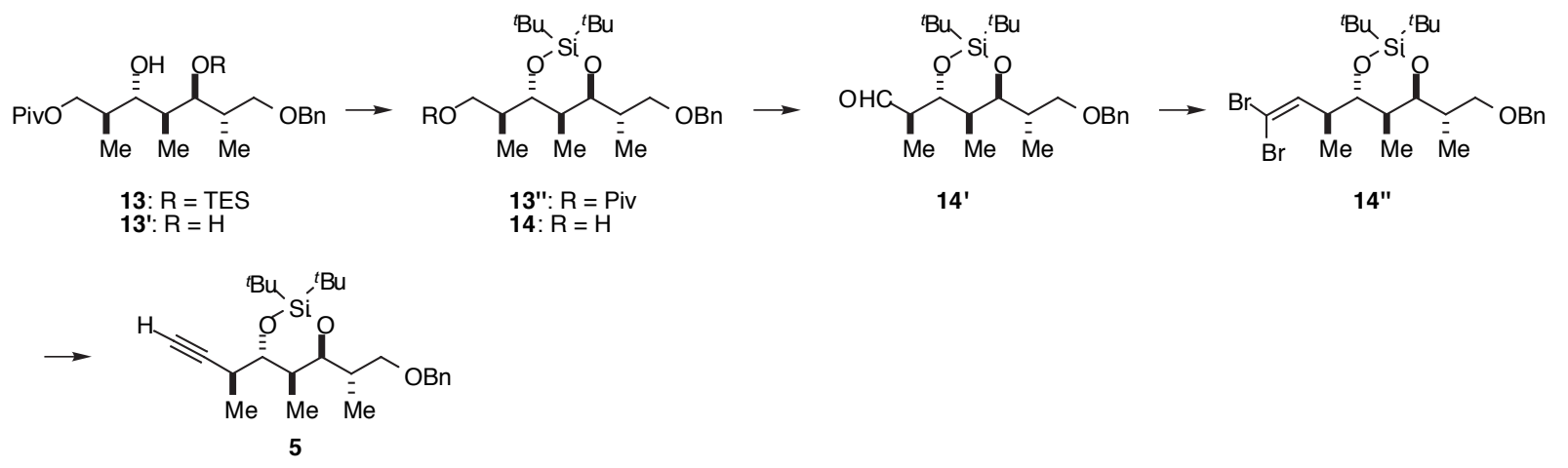

\section{Diol 13'}

To a solution of the crude silyl ether $\mathbf{1 3}^{1}(7.31 \mathrm{mmol})$ in dry THF $(15 \mathrm{~mL})$ was added TBAF $(0.98 \mathrm{M}$ solution in THF, $9.7 \mathrm{~mL}, 9.5 \mathrm{mmol}$ ) at room temperature. After stirring for $1 \mathrm{~h}$, the reaction mixture was quenched with 
$\mathrm{H}_{2} \mathrm{O}$ and THF was removed in vacuo. The residue was extracted with ether (x4). The combined organic layers were washed with brine, dried $\left(\mathrm{MgSO}_{4}\right)$ and concentrated in vacuo. The crude product was purified by silica gel column chromatography (EtOAc/hexane $15 \%)$ to give diol 13' $\left(2.61 \mathrm{~g}, 94 \%\right.$ for 3 steps) as an oil: $[\alpha]_{\mathrm{D}}{ }^{28}=$ $+14.4^{\circ}\left(\mathrm{c} 1.2, \mathrm{CHCl}_{3}\right)$; IR (neat) $3450(\mathrm{br}), 2970,1726,1479,1286,1165,974 \mathrm{~cm}^{-1} ;{ }^{1} \mathrm{H}$ NMR $\delta(400 \mathrm{MHz}$, $\left.\mathrm{CDCl}_{3}\right) 7.39-7.28(5 \mathrm{H}, \mathrm{m}), 4.55(1 \mathrm{H}, \mathrm{d}, J=11.6 \mathrm{~Hz}), 4.51(1 \mathrm{H}, \mathrm{d}, J=11.6 \mathrm{~Hz}), 4.34(1 \mathrm{H}, \mathrm{dd}, J=10.8,4.0$ Hz), $4.28(1 \mathrm{H}, \mathrm{s}), 4.17(1 \mathrm{H}, \mathrm{dd}, J=10.8,6.4 \mathrm{~Hz}), 3.88(1 \mathrm{H}, \mathrm{d}, J=9.0 \mathrm{~Hz}), 3.63-3.59(2 \mathrm{H}, \mathrm{m}), 3.51(1 \mathrm{H}, \mathrm{dd}$, $J=9.2,9.2 \mathrm{~Hz}), 3.34(1 \mathrm{H}, \mathrm{ddd}, J=9.0,8.4,3.6 \mathrm{~Hz}), 2.12-1.99(2 \mathrm{H}, \mathrm{m}), 1.85-1.78(1 \mathrm{H}, \mathrm{m}), 1.21(9 \mathrm{H}, \mathrm{s}), 1.08$ $(3 \mathrm{H}, \mathrm{d}, J=7,2 \mathrm{~Hz}), 0.90(3 \mathrm{H}, \mathrm{d}, J=6.8 \mathrm{~Hz}), 0.77(3 \mathrm{H}, \mathrm{d}, J=7.2 \mathrm{~Hz}) ;{ }^{13} \mathrm{C} \mathrm{NMR} \delta\left(67.8 \mathrm{MHz}, \mathrm{CDCl}_{3}\right) 178.7$, 137.4, 128.5 (2C), 127.9, 127.7 (2C), 77.9, 76.5, 73.6, 67.0, 38.9, 36.8, 35.7, 34.2, 27.2 (3C), 24.7, 14.1, 12.9, 10.7; HRMS $\left(\mathrm{FD}^{+}\right)[\mathrm{M}]^{+}$found 380.2533, $\mathrm{C}_{22} \mathrm{H}_{36} \mathrm{O}_{5}$ requires 380.2563 .

\section{Cyclic silyl ether 13"}

To a solution of diol 13' (1.68 g, $4.43 \mathrm{mmol})$ in dry $\mathrm{CH}_{2} \mathrm{Cl}_{2}(22 \mathrm{~mL})$ was added 2,6-lutidine (2.06 mL, 17.7 mmol) and di-tert-butylsilyl ditriflate $(2.42 \mathrm{~mL}, 6.64 \mathrm{mmol})$ at room temperature. After being stirred for $66 \mathrm{~h}$ at $30{ }^{\circ} \mathrm{C}$, the reaction was diluted with hexane and quenched with $\mathrm{H}_{2} \mathrm{O}$. The mixture was separated and the aqueous layer was extracted with hexane (x3). The combined organic layers were washed with brine, dried $\left(\mathrm{MgSO}_{4}\right)$ and concentrated in vacuo to leave an oil which was purified by silica gel column chromatography (EtOAc/hexane 5\%) to give cyclic silyl ether 13" $(1.7 \mathrm{~g}, 74 \%)$ as an oil: $[\alpha]_{\mathrm{D}}{ }^{26}=-21.8^{\circ}\left(\mathrm{c} 1.3, \mathrm{CHCl}_{3}\right)$; IR (neat) 2970, 2935, 2860, 2360, 1730, 1477, 1285, 1157, 1099, $986 \mathrm{~cm}^{-1} ;{ }^{1} \mathrm{H} \mathrm{NMR} \delta\left(400 \mathrm{MHz}, \mathrm{CDCl}_{3}\right) 7.35-$ $7.27(5 \mathrm{H}, \mathrm{m}), 4.53(1 \mathrm{H}, \mathrm{d}, J=12.0 \mathrm{~Hz}), 4.47(1 \mathrm{H}, \mathrm{d}, J=12.0 \mathrm{~Hz}), 4.23(1 \mathrm{H}, \mathrm{dd}, J=10.4,4.0 \mathrm{~Hz}), 4.09(1 \mathrm{H}$, d, $J=10.4,6.8 \mathrm{~Hz}), 3.97(1 \mathrm{H}, \mathrm{dd}, J=9.6,3.2 \mathrm{~Hz}), 3.69(1 \mathrm{H}, \mathrm{dd}, J=7.6,2.8 \mathrm{~Hz}), 3.67(1 \mathrm{H}, \mathrm{dd}, J=8.8,3.2$ Hz), $3.53(1 \mathrm{H}, \mathrm{dd}, J=7.6,2.4 \mathrm{~Hz}), 2.01-1.84(3 \mathrm{H}, \mathrm{m}), 1.21(9 \mathrm{H}, \mathrm{s}), 1.05(3 \mathrm{H}, \mathrm{d}, J=7.2 \mathrm{~Hz}), 1.03(9 \mathrm{H}, \mathrm{s})$, $1.00(9 \mathrm{H}, \mathrm{s}), 0.97(1 \mathrm{H}, \mathrm{d}, J=6.8 \mathrm{~Hz}), 0.93(1 \mathrm{H}, \mathrm{d}, J=6.8 \mathrm{~Hz}) ;{ }^{13} \mathrm{C} \mathrm{NMR} \delta\left(100.4 \mathrm{MHz}, \mathrm{CDCl}_{3}\right) 178.4,138.7$, 128.1 (2C), 127.6 (2C), 127.2, 80.8, 73.1, 73.0, 72.4, 66.2, 39.5, 38.9, 37.1, 35.7, 28.3 (3C), 27.8 (3C), 27.3 (3C), 22.1, 21.8, 13.9, 13.8, 13.8; HRMS $\left(\mathrm{FD}^{+}\right)[\mathrm{M}]^{+}$found 520.3563, $\mathrm{C}_{30} \mathrm{H}_{52} \mathrm{O}_{5}$ Si requires 520.3584.

\section{Alcohol 14}

To a solution of pivalate 13" (1.95 g, $3.75 \mathrm{mmol})$ in dry THF (15 mL) was added DIBAL (0.95 $M$ solution in hexane, $8.7 \mathrm{~mL}, 8.25 \mathrm{mmol}$ ) at $0{ }^{\circ} \mathrm{C}$. After stirring for $30 \mathrm{~min}$, the reaction mixture was quenched with satd. aq. potassium sodium tartrate solution, and then the mixture was separated. The organic layer was washed with brine and the aqueous layers were extracted with ether (x4). The combined organic layers were dried $\left(\mathrm{MgSO}_{4}\right)$ and concentrated in vacuo. The crude product was purified by silica gel column chromatography (EtOAc/hexane 5\%, 10\%) to give alcohol $14(1.51 \mathrm{~g}, 92 \%)$ as an oil: $[\alpha]_{\mathrm{D}}{ }^{26}=-17.9^{\circ}$ (c 1.3, $\left.\mathrm{CHCl}_{3}\right)$; IR (neat) 3450 (br), 2968, 2956, 2860, 1475, 1097, 986, $756 \mathrm{~cm}^{-1}$; ${ }^{1} \mathrm{H}$ NMR $\delta$ (400 MHz, $\mathrm{CDCl}_{3}$ ) 7.35-7.27 (5 H, m), 4.54 
$(1 \mathrm{H}, \mathrm{d}, J=11.6 \mathrm{~Hz}), 4.46(1 \mathrm{H}, \mathrm{d}, J=11.6 \mathrm{~Hz}), 4.05(1 \mathrm{H}, \mathrm{dd}, J=10.0,3.2 \mathrm{~Hz}), 3.75(1 \mathrm{H}, \mathrm{dd}, J=7.2,3.6 \mathrm{~Hz})$, $3.72(1 \mathrm{H}, \mathrm{dd}, J=7.2,3.6 \mathrm{~Hz}), 3.67(1 \mathrm{H}, \mathrm{ddd}, J=6.8,4.0,3.2 \mathrm{~Hz}), 3.65(1 \mathrm{H}, \mathrm{ddd}, J=7.2,6.8,4.0 \mathrm{~Hz}), 3.56$ $(1 \mathrm{H}, \mathrm{dd}, J=8.4,6.0 \mathrm{~Hz}), 3.16(1 \mathrm{H}, \mathrm{dd}, J=7.2,4.0 \mathrm{~Hz}), 1.94-1.78(3 \mathrm{H}, \mathrm{m}), 1.06(9 \mathrm{H}, \mathrm{s}), 1.04(3 \mathrm{H}, \mathrm{d}, J=7.2$ $\mathrm{Hz}), 1.02(9 \mathrm{H}, \mathrm{s}), 0.94(3 \mathrm{H}, \mathrm{d}, J=6.8 \mathrm{~Hz}), 0.89(3 \mathrm{H}, \mathrm{d}, J=6.8 \mathrm{~Hz}) ;{ }^{13} \mathrm{C} \mathrm{NMR} \delta\left(100.4 \mathrm{MHz}, \mathrm{CDCl}_{3}\right) 138.6$, 128.1 (2C), 127.5 (2C), 127.3, 85.4, 73.1, 72.9, 72.3, 67.6, 41.5, 37.3, 37.0, 28.4 (3C), 27.7 (3C), 21.9, 21.8, 13.8, 13.8, 13.8; HRMS (FD+) [M+H]+ found 437.3067, $\mathrm{C}_{25} \mathrm{H}_{45} \mathrm{O}_{4} \mathrm{Si}$ requires 437.3087.

\section{Aldehyde 14'}

To a solution of oxalyl chloride $(0.36 \mathrm{~mL}, 4.07 \mathrm{mmol})$ in dry $\mathrm{CH}_{2} \mathrm{Cl}_{2}(5 \mathrm{~mL})$ was added dropwise DMSO $(0.58 \mathrm{~mL}, 8.15 \mathrm{mmol})$ at $-78{ }^{\circ} \mathrm{C}$ and the mixture was stirred for $10 \mathrm{~min}$. After a solution of alcohol 14 in $\mathrm{CH}_{2} \mathrm{Cl}_{2}$ $(5 \mathrm{~mL})$ was added to the mixture, stirring was continued at $-78{ }^{\circ} \mathrm{C}$ for $20 \mathrm{~min}$, then triethylamine $(1.7 \mathrm{~mL}, 12.2$ mmol) was added, and the mixture was further stirred at room temperature for $30 \mathrm{~min}$. The reaction mixture was treated with $\mathrm{H}_{2} \mathrm{O}$ and separated. The aqueous layers were extracted with hexane (x4). The combined organic layers were dried $\left(\mathrm{MgSO}_{4}\right)$ and concentrated in vacuo to give the aldehyde 14' which was used for the next step without purification: ${ }^{1} \mathrm{H}$ NMR $\delta\left(270 \mathrm{MHz}, \mathrm{CDCl}_{3}\right) 9.75(1 \mathrm{H}, \mathrm{d}, J=3.5 \mathrm{~Hz}), 7.36-7.22(5 \mathrm{H}, \mathrm{m}), 4.54(1 \mathrm{H}, \mathrm{d}, J$ $=11.9 \mathrm{~Hz}), 4.46(1 \mathrm{H}, \mathrm{d}, J=11.8 \mathrm{~Hz}), 4.05(1 \mathrm{H}, \mathrm{dd}, J=7.8,5.0 \mathrm{~Hz}), 4.03-4.00(1 \mathrm{H}, \mathrm{m}), 3.65(1 \mathrm{H}, \mathrm{dd}, J=8.5$, $3.1 \mathrm{~Hz}), 3.55(1 \mathrm{H}, \mathrm{dd}, J=8.5,6.0 \mathrm{~Hz}), 2.63-2.48(1 \mathrm{H}, \mathrm{m}), 1.96-1.81(2 \mathrm{H}, \mathrm{m}), 1.10(3 \mathrm{H}, \mathrm{d}, J=7.4 \mathrm{~Hz}), 1.07$ (3H, d, $J=6.9 \mathrm{~Hz}), 1.03(9 \mathrm{H}, \mathrm{s}), 0.98(9 \mathrm{H}, \mathrm{s}), 0.92(3 \mathrm{H}, \mathrm{d}, J=6.9 \mathrm{~Hz})$.

\section{Dibromo olefin 14"}

To a mixture of triphenylphosphine $(2.68 \mathrm{~g}, 10.2 \mathrm{mmol})$ and tetrabromomethane $(1.35 \mathrm{~g}, 4.08 \mathrm{mmol})$ in dry $\mathrm{CH}_{2} \mathrm{Cl}_{2}(5 \mathrm{~mL})$ was added pyridine $(0.99 \mathrm{~mL}, 12.2 \mathrm{mmol})$ at $0{ }^{\circ} \mathrm{C}$ and the reaction mixture was stirred for $5 \mathrm{~min}$. After addition of a solution of the crude aldehyde $\mathbf{1 4}^{\prime}$ in $\mathrm{CH}_{2} \mathrm{Cl}_{2}(5 \mathrm{~mL})$ at $0{ }^{\circ} \mathrm{C}$, the mixture was stirred for 30 min, then quenched with satd. $\mathrm{NaHCO}_{3}$ solution, and separated. The aqueous layers were extracted with $\mathrm{CH}_{2} \mathrm{Cl}_{2}$ (x3). The combined organic layers were dried $\left(\mathrm{MgSO}_{4}\right)$ and concentrated in vacuo. The crude product was purified by silica gel column chromatography (EtOAc/hexane 1\%) to give 14” (1.15 g, 96\% from 14) as a white solid: $[\alpha]_{\mathrm{D}}{ }^{25}=-3.8^{\circ}\left(\mathrm{c} 1.2, \mathrm{CHCl}_{3}\right) ; \mathrm{mp} 47-52{ }^{\circ} \mathrm{C}$; IR (neat) 2968, 2933, 2860, 1475, $1007 \mathrm{~cm}^{-1} ;{ }^{1} \mathrm{H}$ NMR $\delta(400$ $\left.\mathrm{MHz}, \mathrm{CDCl}_{3}\right)$ 7.36-7.23 (5 H, m), $6.55(1 \mathrm{H}, \mathrm{d}, J=9.6 \mathrm{~Hz}), 4.53(1 \mathrm{H}, \mathrm{d}, J=12.0 \mathrm{~Hz}), 4.48(1 \mathrm{H}, \mathrm{d}, J=12.0$ $\mathrm{Hz}), 3.89(1 \mathrm{H}, \mathrm{dd}, J=9.6,4.0 \mathrm{~Hz}), 3.73(1 \mathrm{H}, \mathrm{dd}, J=4.0,4.0 \mathrm{~Hz}), 3.71(1 \mathrm{H}, \mathrm{dd}, J=8.8,3.2 \mathrm{~Hz}), 3.49(1 \mathrm{H}, \mathrm{dd}$, $J=8.8,2.8 \mathrm{~Hz}), 2.54(1 \mathrm{H}, \mathrm{dqd}, J=9.6,6.8,4.0 \mathrm{~Hz}), 1.90(1 \mathrm{H}, \mathrm{qddd}, J=6.8,4.0,3.2,2.8 \mathrm{~Hz}), 1.86(1 \mathrm{H}$, dqd, $J=9.6,7.2,4.0 \mathrm{~Hz}), 1.08(3 \mathrm{H}, \mathrm{d}, J=6.8 \mathrm{~Hz}), 1.03(9 \mathrm{H}, \mathrm{s}), 1.02(3 \mathrm{H}, \mathrm{d}, J=7.2 \mathrm{~Hz}), 1.01(9 \mathrm{H}, \mathrm{s}), 0.95(3 \mathrm{H}, \mathrm{d}$, $J=6.8 \mathrm{~Hz}) ;{ }^{13} \mathrm{C} \mathrm{NMR} \delta\left(67.8 \mathrm{MHz}, \mathrm{CDCl}_{3}\right)$ 140.6, 138.7, 128.1 (2C), 127.5 (2C), 127.2, 88.0, 81.6, 73.6, 73.0, 72.5, 45.2, 37.3, 36.8, 28.3 (3C), 27.7 (3C), 22.2, 21.9, 16.1, 14.1, 13.5; HRMS (FD $)[\mathrm{M}]^{+}$found 588.1245, $\mathrm{C}_{26} \mathrm{H}_{42} \mathrm{Br}_{2} \mathrm{O}_{3}$ Si requires 588.1270. 


\section{Acetylene 5}

To a solution of 14"' (939 mg, $1.59 \mathrm{mmol})$ in dry THF $(8 \mathrm{~mL})$ was added $n \mathrm{BuLi}(1.29 \mathrm{M}$ solution in hexane, $2.7 \mathrm{~mL}, 3.5 \mathrm{mmol}$ ) at $-30{ }^{\circ} \mathrm{C}$. After stirring for $30 \mathrm{~min}$, the reaction mixture was quenched with satd. $\mathrm{NH}_{4} \mathrm{Cl}$ and $\mathrm{H}_{2} \mathrm{O}$, and then the mixture was separated. The aqueous layers were extracted with ether $(\mathrm{x} 4)$. The combined organic layers were dried $\left(\mathrm{MgSO}_{4}\right)$ and concentrated in vacuo. The crude product was purified by silica gel column chromatography (EtOAc/hexane $0.5 \%)$ to give acetylene $5(617.7 \mathrm{mg}, 90 \%)$ as an oil: $[\alpha]_{\mathrm{D}}{ }^{28}=-26.8^{\circ}(\mathrm{c}$ 1.4, $\mathrm{CHCl}_{3}$ ); IR (neat) 3311. 2970, 2858, 1477, $982 \mathrm{~cm}^{-1} ;{ }^{1} \mathrm{H} \mathrm{NMR} \delta\left(400 \mathrm{MHz}, \mathrm{CDCl}_{3}\right)$ 7.34-7.25 (5 H, m), $4.53(1 \mathrm{H}, \mathrm{d}, J=12.0 \mathrm{~Hz}), 4.48(1 \mathrm{H}, \mathrm{d}, J=12.0 \mathrm{~Hz}), 4.05(1 \mathrm{H}, \mathrm{dd}, J=9.6,3.6 \mathrm{~Hz}), 3.76(1 \mathrm{H}, \mathrm{dd}, J=4.0,3.6$ $\mathrm{Hz}), 3.74(1 \mathrm{H}, \mathrm{dd}, J=8.8,3.2 \mathrm{~Hz}), 3.47(1 \mathrm{H}, \mathrm{dd}, J=8.8,2.8 \mathrm{~Hz}), 2.62(1 \mathrm{H}, \mathrm{qdd}, J=6.8,3.6,2.4 \mathrm{~Hz}), 2.11(1$ $\mathrm{H}$, qddd, $J=7.2,3.6,3.2,2.8 \mathrm{~Hz}), 1.99(1 \mathrm{H}, \mathrm{d}, J=2.4 \mathrm{~Hz}), 1.91(1 \mathrm{H}$, dqd, $J=9.6,6.8,4.0 \mathrm{~Hz}), 1.24(3 \mathrm{H}, \mathrm{d}, J$ $=6.8 \mathrm{~Hz}), 1.05(3 \mathrm{H}, \mathrm{d}, J=7.2 \mathrm{~Hz}), 1.04(18 \mathrm{H}, \mathrm{s}), 0.96(3 \mathrm{H}, \mathrm{d}, J=6.8 \mathrm{~Hz}) ;{ }^{13} \mathrm{C} \mathrm{NMR} \delta\left(67.8 \mathrm{MHz}, \mathrm{CDCl}_{3}\right)$ 138.8, 128.2 (2C), 127.6 (2C), 127.3, 86.0, 80.1, 73.9, 73.0, 72.6, 69.4, 37.0, 36.8, 33.6, 28.2 (3C), 27.7 (3C), 22.2, 21.9, 16.6, 13.9, 13.9; HRMS (FD+) [M]+ found 430.2884, $\mathrm{C}_{26} \mathrm{H}_{42} \mathrm{O}_{3}$ Si requires 430.2903.

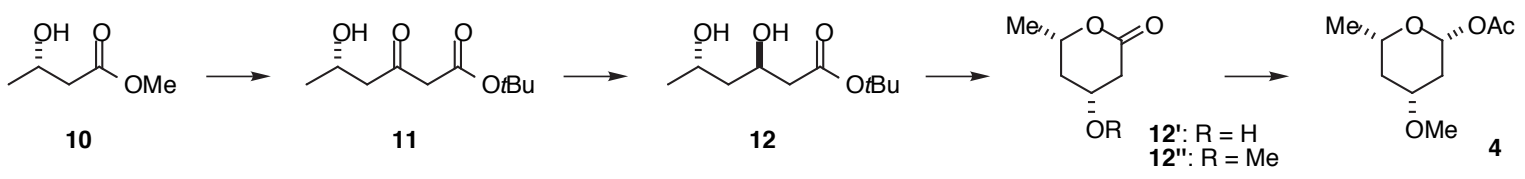

\section{Ester 11}

To a solution of LDA prepared from $i \mathrm{Pr}_{2} \mathrm{NH}(21 \mathrm{~mL}, 150 \mathrm{mmol})$ and $n \mathrm{BuLi}$ (2.64 $M$ solution in hexane, 45.5 $\mathrm{mL}, 120 \mathrm{mmol})$ in THF $(150 \mathrm{~mL})$ by stirring for $15 \mathrm{~min}$ at $0{ }^{\circ} \mathrm{C}$ was added tert-butyl acetate $(16.2 \mathrm{~mL}, 120$ mmol) at $-78{ }^{\circ} \mathrm{C}$ and the reaction mixture was stirred for $20 \mathrm{~min}$. After dropwise addition of a solution of methyl (S)-3-hydroxybutanoate $(\mathbf{1 0})$ in THF $(15 \mathrm{~mL})$ at $-78{ }^{\circ} \mathrm{C}$, the mixture was stirred for $2 \mathrm{~h}$ at $-50{ }^{\circ} \mathrm{C}$, and then stirred for $15 \mathrm{~min}$ at $-15{ }^{\circ} \mathrm{C}$. The reaction mixture was quenched with ice cold $\mathrm{H}_{2} \mathrm{O}$, and separated. The aqueous layers were extracted with ether (x3). The combined organic layers were washed with satd. $\mathrm{NaHCO}_{3}$ solution and brine, then dried $\left(\mathrm{MgSO}_{4}\right)$ and concentrated in vacuo. The crude product was purified by silica gel column chromatography (EtOAc/hexane $20 \%, 25 \%)$ to give ester $11(5.64 \mathrm{~g}, 93 \%)$ as an oil: $[\alpha]_{\mathrm{D}}^{28}=+38.7^{\circ}(\mathrm{c}$ 1.1, $\left.\mathrm{CHCl}_{3}\right)$; IR (neat) 3450 (br), 2978, 1711, $1150 \mathrm{~cm}^{-1} ;{ }^{1} \mathrm{H}$ NMR $\delta\left(400 \mathrm{MHz}, \mathrm{CDCl}_{3}\right) 4.26(1 \mathrm{H}, \mathrm{dqdd}, J=8.8$, 6.4, 3.2, 3.2 Hz), $3.37(2 \mathrm{H}, \mathrm{s}), 2.86(1 \mathrm{H}, \mathrm{d}, J=3.2 \mathrm{~Hz}), 2.74(1 \mathrm{H}, \mathrm{dd}, J=17.6,3.2 \mathrm{~Hz}), 2.64(1 \mathrm{H}, \mathrm{dd}, J=17.6$, $8.8 \mathrm{~Hz}), 1.47(9 \mathrm{H}, \mathrm{s}), 1.21(3 \mathrm{H}, \mathrm{d}, J=6.4 \mathrm{~Hz}) ;{ }^{13} \mathrm{C} \mathrm{NMR} \delta\left(67.8 \mathrm{MHz}, \mathrm{CDCl}_{3}\right)$ 204.0, 166.1, 82.1, 63.6, 51.0, 50.9, $27.8(3 \mathrm{C}), 22.3$; HRMS $\left(\mathrm{FD}^{+}\right)[\mathrm{M}+\mathrm{H}]^{+}$found 203.1283, $\mathrm{C}_{10} \mathrm{H}_{19} \mathrm{O}_{4}$ requires 203.1283.

\section{Dihydroxy ester 12}

To a solution of $\mathrm{Me}_{4} \mathrm{NBH}(\mathrm{OAc})_{3}(30.4 \mathrm{~g}, 115.5 \mathrm{mmol})$ in dry $\mathrm{MeCN}(91 \mathrm{~mL})$ was added $\mathrm{AcOH}(91 \mathrm{~mL})$ at 
room temperature and the reaction mixture was stirred for $30 \mathrm{~min}$. After addition of a solution of $\beta$-ketoester 11 (3.68 g, $18.2 \mathrm{mmol})$ in $\mathrm{MeCN}(27 \mathrm{~mL})$ at $-40{ }^{\circ} \mathrm{C}$, the mixture was stirred for $94 \mathrm{~h}$, then quenched with satd. aq. potassium sodium tartrate solution, and separated. The aqueous layers were extracted with $\mathrm{CH}_{2} \mathrm{Cl}_{2}(\mathrm{x} 4)$ and the combined organic layers were washed with satd. $\mathrm{NaHCO}_{3}$ solution and brine, dried $\left(\mathrm{MgSO}_{4}\right)$, and concentrated in vacuo. The crude product was purified by silica gel column chromatography (EtOAc/hexane 25\%, 30\%, 35\%, $40 \%, 45 \%)$ to give the dihydroxy ester $12(3.69 \mathrm{~g}, 97 \%)$ as an oil: $[\alpha]_{\mathrm{D}}{ }^{19}=-4.7^{\circ}\left(\mathrm{c} 1.4, \mathrm{CHCl}_{3}\right)$; IR (neat) 3395 (br), 2976, 2934, 1728, 1369, $1155 \mathrm{~cm}^{-1} ;{ }^{1} \mathrm{H} \mathrm{NMR} \delta\left(400 \mathrm{MHz}, \mathrm{CDCl}_{3}\right) 4.31$ (1H, ddddd, $J=8.8,8.3,3.7,3.7$, $2.7 \mathrm{~Hz}), 4.18-4.08(1 \mathrm{H}, \mathrm{m}), 3.57(1 \mathrm{H}, \mathrm{d}, J=2.7 \mathrm{~Hz}), 2.63-2.57(1 \mathrm{H}, \mathrm{m}), 2.46(1 \mathrm{H}, \mathrm{dd}, J=16.5,8.8 \mathrm{~Hz}), 2.39$ $(1 \mathrm{H}, \mathrm{dd}, J=16.5,3.7 \mathrm{~Hz}), 1.68-1.53(2 \mathrm{H}, \mathrm{m}), 1.47(9 \mathrm{H}, \mathrm{s}), 1.24(3 \mathrm{H}, \mathrm{d}, J=6.3 \mathrm{~Hz}) ;{ }^{13} \mathrm{C}$ NMR $\delta(67.8 \mathrm{MHz}$, $\left.\mathrm{CDCl}_{3}\right)$ 172.1, 81.1, 65.5, 64.5, 43.7, 42.4, 27.9 (3C), 23.4; HRMS (EI $\left.{ }^{+}\right)\left[\mathrm{M}-\mathrm{O}^{t} \mathrm{Bu}\right]^{+}$found 131.0718, $\mathrm{C}_{6} \mathrm{H}_{11} \mathrm{O}_{3}$ requires 131.0708 .

\section{Lactone 12,}

A mixture of the dihydroxy ester $12(2.01 \mathrm{~g}, 9.86 \mathrm{mmol})$ and PPTS (247.8 $\mathrm{mg}, 0.986 \mathrm{mmol})$ in dry $\mathrm{ClCH}_{2} \mathrm{CH}_{2} \mathrm{Cl}(50 \mathrm{~mL})$ was refluxed for $4 \mathrm{~h}$ and then concentrated. The residue was filtered through a pad of silica gel by the aid of AcOEt and the filtrate was concentrated in vacuo to give lactone 12' which was used for the next step without purification: $[\alpha]_{\mathrm{D}}{ }^{19}=-26.2^{\circ}\left(\mathrm{c} 1.4, \mathrm{CHCl}_{3}\right)$; IR (neat) 3410 (br), 2982, 2936, 1728, 1389, 1254, $1078 \mathrm{~cm}^{-1} ;{ }^{1} \mathrm{H}$ NMR $\delta\left(400 \mathrm{MHz}, \mathrm{CDCl}_{3}\right) 4.35(1 \mathrm{H}, \mathrm{dqd}, J=11.5,6.3,2.9 \mathrm{~Hz}), 4.30-4.21(1 \mathrm{H}, \mathrm{m}), 2.91$ $(1 \mathrm{H}, \mathrm{dddd}, J=17.1,5.9,1.5,0.7 \mathrm{~Hz}), 2.46(1 \mathrm{H}, \mathrm{dd}, J=17.1,8.1 \mathrm{~Hz}), 2.27(1 \mathrm{H}, \mathrm{dddd}, J=13.7,5.4,2.9,1.5 \mathrm{~Hz})$, 2.14-2.05 (1H, m), $1.59(1 \mathrm{H}, \mathrm{ddd}, J=13.7,11.5,9.3 \mathrm{~Hz}), 1.42(3 \mathrm{H}, \mathrm{d}, J=6.3 \mathrm{~Hz}) ;{ }^{13} \mathrm{C}$ NMR $\delta(67.8 \mathrm{MHz}$, $\left.\mathrm{CDCl}_{3}\right)$ 171.8, 73.9, 63.2, 39.1, 39.0, 21.2; HRMS $\left(\mathrm{EI}^{+}\right)[\mathrm{M}]^{+}$found 130.0644, $\mathrm{C}_{6} \mathrm{H}_{10} \mathrm{O}_{3}$ requires 131.0630.

\section{Methl ether 12"}

To a mixture of powdered molecular sieves $4 \mathrm{~A}(22.8 \mathrm{~g})$ and $\mathrm{Ag}_{2} \mathrm{O}(15.1 \mathrm{~g}, 65.2 \mathrm{mmol})$ in ether was added MeI (18.4 mL, $295.8 \mathrm{mmol})$ at room temperature and the reaction mixture was stirred for $10 \mathrm{~min}$. After addition of a solution of the crude lactone 12' in dry $\mathrm{CH}_{2} \mathrm{Cl}_{2}$, the mixture was stirred for $1.5 \mathrm{~h}$ with ultra sound, then filtered through a pad of Celite by the aid of $\mathrm{CH}_{2} \mathrm{Cl}_{2}$ and the filtrate was concentrated in vacuo. The residue was purified by neutral silica gel column chromatography (EtOAc/hexane 50\%) to give methyl ether 12” (1.22 g, $86 \%$ ) as an oil: $[\alpha]_{\mathrm{D}}{ }^{19}=-19.7^{\circ}\left(\mathrm{c} 1.4, \mathrm{CHCl}_{3}\right)$; IR (neat) 2982, 2936, 1740, 1389, 1256, 1065, $980 \mathrm{~cm}^{-1} ;{ }^{1} \mathrm{H}$ NMR $\delta\left(400 \mathrm{MHz}, \mathrm{CDCl}_{3}\right) 4.33(1 \mathrm{H}, \mathrm{dqd}, J=11.7,6.2,2.8 \mathrm{~Hz}), 3.75(1 \mathrm{H}, \mathrm{ddt}, J=8.9,7.3,5.9 \mathrm{~Hz}), 3.36(3 \mathrm{H}$, s), $2.86(1 \mathrm{H}, \mathrm{ddd}, J=17.1,5.9,1.2 \mathrm{~Hz}), 2.50(1 \mathrm{H}, \mathrm{dd}, J=17.1,7.3 \mathrm{~Hz}), 2.31(1 \mathrm{H}, \mathrm{dddd}, J=13.9,5.9,2.8,1.2$ $\mathrm{Hz}), 1.52(1 \mathrm{H}, \mathrm{ddd}, J=13.9,11.7,8.9 \mathrm{~Hz}), 1.42(3 \mathrm{H}, \mathrm{d}, J=6.2 \mathrm{~Hz}) ;{ }^{13} \mathrm{C}$ NMR $\delta\left(67.8 \mathrm{MHz}, \mathrm{CDCl}_{3}\right) 170.3$, 73.1, 72.0, 55.6, 36.2, 35.7, 21.1; HRMS (FD ${ }^{+}$[ $[\mathrm{M}]^{+}$found 144.0770, $\mathrm{C}_{7} \mathrm{H}_{12} \mathrm{O}_{3}$ requires 144.0786.

\section{Acetate 4}


To a solution of lactone 12" (2.98 g, $20.7 \mathrm{mmol})$ in dry $\mathrm{CH}_{2} \mathrm{Cl}_{2}(140 \mathrm{~mL})$ was added DIBAL (1.01 $M$ solution in toluene, $24.6 \mathrm{~mL}, 24.8 \mathrm{mmol}$ ) at $-78{ }^{\circ} \mathrm{C}$ and the reaction mixture was stirred for $1 \mathrm{~h}$. After addition of pyridine $(5 \mathrm{~mL}, 62.1 \mathrm{mmol})$, DMAP $(3 \mathrm{~g}, 24.8 \mathrm{mmol})$ and acetic anhydride $(7.8 \mathrm{~mL}, 82.8 \mathrm{mmol})$ at $-78{ }^{\circ} \mathrm{C}$, the mixture was stirred for $1 \mathrm{~h}$ at room temperature, and then quenched with satd. aq. potassium sodium tartrate solution, and the mixture was separated. The organic layer was washed with brine and the aqueous layers were extracted with ether (x4). The combined organic layers were dried $\left(\mathrm{MgSO}_{4}\right)$ and concentrated in vacuo. The crude product was purified by silica gel column chromatography (EtOAc/hexane 20\%) to give acetate $4(3.11 \mathrm{~g}$, $80 \%$ ) as an oil: $[\alpha]_{\mathrm{D}}{ }^{30}=+5.8^{\circ}(\mathrm{c} 1.1, \mathrm{CHCl} 3)$; IR (neat) $2976,2937,1753,1375,1231,1148,1047 \mathrm{~cm}^{-1}$; ${ }^{1} \mathrm{H}$ NMR $\delta\left(400 \mathrm{MHz}, \mathrm{CDCl}_{3}\right) 5.64(1 \mathrm{H}, \mathrm{dd}, J=10.4,2.0 \mathrm{~Hz}), 3.61(1 \mathrm{H}, \mathrm{dqd}, J=11.6,6.0,2.0 \mathrm{~Hz}), 3.44(1 \mathrm{H}$, dddd, $J=11.2,11.2,4.8,4.4 \mathrm{~Hz}), 3.36(3 \mathrm{H}, \mathrm{s}), 2.25$ (1 H, dddd, $J=12.0,4.4,2.4,2.0 \mathrm{~Hz}), 2.12(3 \mathrm{H}, \mathrm{s}), 2.01$ (1 H, dddd, $J=12.8,4.8,2.4,2.0 \mathrm{~Hz}), 1.40(1 \mathrm{H}, \mathrm{ddd}, J=12.0,11.2,10.4 \mathrm{~Hz}), 1.29(3 \mathrm{H}, \mathrm{d}, J=6.0 \mathrm{~Hz}), 1.18(1$ $\mathrm{H}$, ddd, $J=12.8,11.6,11.2 \mathrm{~Hz}) ;{ }^{13} \mathrm{C} \mathrm{NMR} \delta\left(67.8 \mathrm{MHz}, \mathrm{CDCl}_{3}\right)$ 169.0, 92.5, 74.7, 69.1, 55.4, 38.2, 35.9, 21.1, 21.0; HRMS $\left(\mathrm{FD}^{+}\right)[\mathrm{M}]^{+}$found 188.1028, $\mathrm{C}_{9} \mathrm{H}_{16} \mathrm{O}_{4}$ requires 188.1049 .
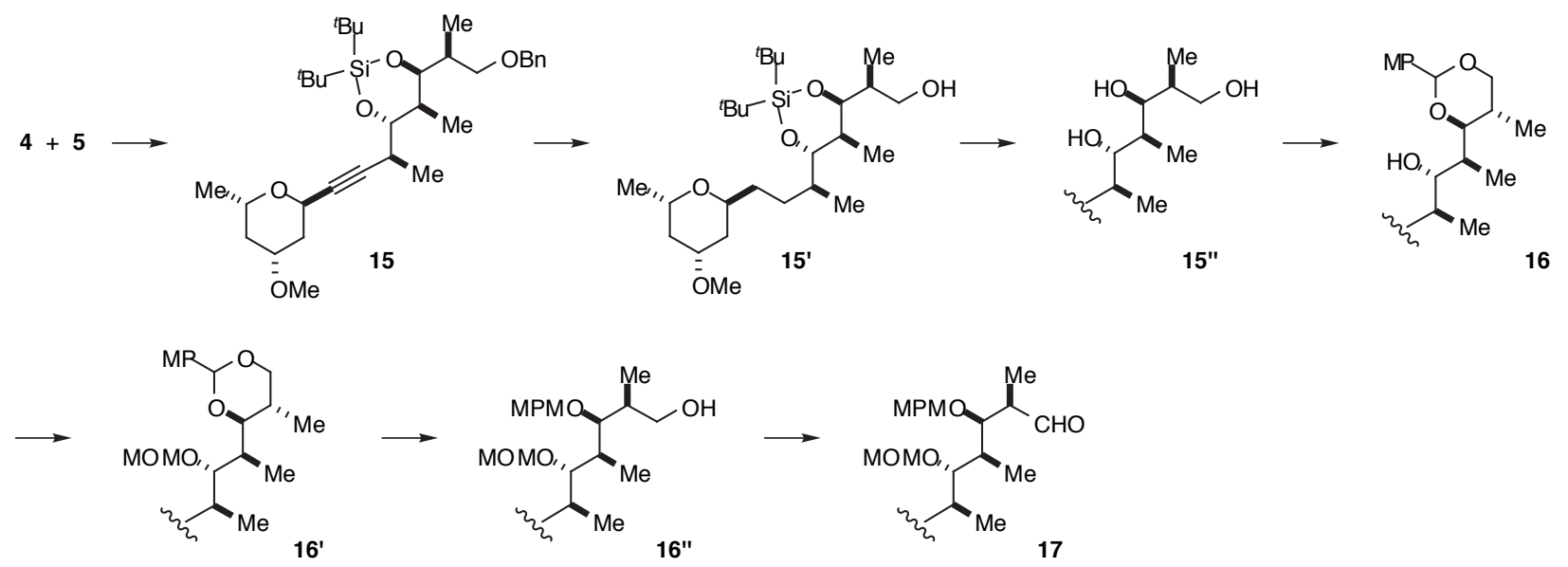

\section{Coupling compound 15}

To a solution of $4(1.29 \mathrm{~g}, 3.0 \mathrm{mmol})$ in dry $\mathrm{CH}_{2} \mathrm{Cl}_{2}(7 \mathrm{~mL})$ was added $n \mathrm{BuLi}(1 \mathrm{M}$ solution in hexane, $3 \mathrm{~mL}$, $3 \mathrm{mmol}$ ) at $-30{ }^{\circ} \mathrm{C}$ and the mixture was stirred for $30 \mathrm{~min}$. After addition of $\mathrm{Me}_{2} \mathrm{AlOTf}\left(1 \mathrm{M}\right.$ solution in $\mathrm{CH}_{2} \mathrm{Cl}_{2}$,

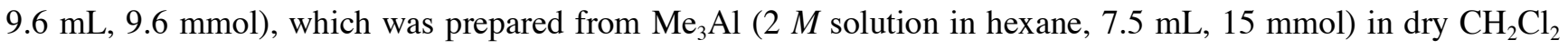
$(7.5 \mathrm{~mL})$ and $\mathrm{TfOH}(1.33 \mathrm{~mL}, 15 \mathrm{mmol})$ by stirring for $20 \mathrm{~min}$ at $0{ }^{\circ} \mathrm{C}$, at $-30{ }^{\circ} \mathrm{C}$, the mixture was stirred for 30 min. To the resulting alkynyl aluminum solution was added a solution of 5 (702 $\mathrm{mg}, 3.73 \mathrm{mmol})$ in $\mathrm{CH}_{2} \mathrm{Cl}_{2}(8$ $\mathrm{mL}$ ) at $-30{ }^{\circ} \mathrm{C}$ and the mixture was stirred for $30 \mathrm{~min}$ at $-30{ }^{\circ} \mathrm{C}$, then stirred for $30 \mathrm{~min}$ at room temperature. The reaction was quenched with satd. aq. potassium sodium tartrate solution and $\mathrm{NaHCO}_{3}$, and the mixture was separated. The organic layer was washed with brine and the aqueous layers were extracted with hexane (x4). 
The combined organic layers were dried $\left(\mathrm{MgSO}_{4}\right)$ and concentrated in vacuo. The crude product was purified by silica gel column chromatography (EtOAc/hexane 2\%, 5\%) to give the coupling compound 15 (1.33 g, 80\%) as an oil: $[\alpha]_{\mathrm{D}}^{28}=-35.6^{\circ}\left(\mathrm{c} 1.0, \mathrm{CHCl}_{3}\right)$; IR (neat) 2970, 2934, 2860, 1097, $756 \mathrm{~cm}^{-1} ;{ }^{1} \mathrm{H} \mathrm{NMR} \delta(400 \mathrm{MHz}$, $\left.\mathrm{CDCl}_{3}\right)$ 7.35-7.25 (5 H, m), 4.84-4.80 (1 H, m), $4.52(1 \mathrm{H}, \mathrm{d}, J=12.0 \mathrm{~Hz}), 4.46(1 \mathrm{H}, \mathrm{d}, J=12.0 \mathrm{~Hz}), 4.03(1 \mathrm{H}$, dd, $J=9.6,3.2 \mathrm{~Hz}), 3.99(1 \mathrm{H}, \mathrm{dqd}, J=11.6,6.0,2.0 \mathrm{~Hz}), 3.74(1 \mathrm{H}, \mathrm{dd}, J=4.4,4.0 \mathrm{~Hz}), 3.70(1 \mathrm{H}, \mathrm{dd}, J=8.4$, $3.2 \mathrm{~Hz}), 3.65$ (1 H, dddd, $J=11.2,10.8,4.4,4.4 \mathrm{~Hz}), 3.50(1 \mathrm{H}, \mathrm{dd}, J=8.4,6.4 \mathrm{~Hz}), 3.31(3 \mathrm{H}, \mathrm{s}), 2.67(1 \mathrm{H}$, qdd, $J=6.8,3.2,2.0 \mathrm{~Hz}), 2.08(1 \mathrm{H}, \mathrm{qddd}, J=7.2,6.4,4.0,3.2 \mathrm{~Hz}), 2.04(1 \mathrm{H}, \mathrm{dddd}, J=12.4,4.4,2.4,2.0 \mathrm{~Hz})$, $1.98(1 \mathrm{H}, \mathrm{dddd}, J=12.4,4.4,2.4,2.0 \mathrm{~Hz}), 1.90(1 \mathrm{H}, \mathrm{dqd}, J=9.6,6.8,4.4 \mathrm{~Hz}), 1.53(1 \mathrm{H}, \mathrm{ddd}, J=12.4,11.2$, $5.6 \mathrm{~Hz}), 1.23(3 \mathrm{H}, \mathrm{d}, J=6.8 \mathrm{~Hz}), 1.18(3 \mathrm{H}, \mathrm{d}, J=6.0 \mathrm{~Hz}), 1.09(1 \mathrm{H}, \mathrm{ddd}, J=12.4,11.6,10.8 \mathrm{~Hz}), 1.04(3 \mathrm{H}$, $\mathrm{d}, J=7.2 \mathrm{~Hz}), 1.04(18 \mathrm{H}, \mathrm{s}), 0.96(3 \mathrm{H}, \mathrm{d}, J=6.8 \mathrm{~Hz}) ;{ }^{13} \mathrm{C} \mathrm{NMR} \delta\left(100.4 \mathrm{MHz}, \mathrm{CDCl}_{3}\right)$ 138.6, $128.1(2 \mathrm{C})$, 127.6 (2C), 127.3, 88.5, 80.2, 79.3, 73.9, 73.4, 73.1, 72.4, 66.6, 64.6, 55.2, 39.6, 37.0, 36.9, 36.4, 33.7, 28.2 (3C), 27.8 (3C), 22.1, 21.9, 21.8, 16.9, 14.0, 13.9; HRMS (FD+) [M]+ found 558.3748, $\mathrm{C}_{33} \mathrm{H}_{54} \mathrm{O}_{5} \mathrm{Si}$ requires 558.3741 .

\section{Alcohol 15,}

A mixture of 15 (283.9 mg, $0.51 \mathrm{mmol})$ and $10 \%$ palladium hydroxide on carbon (28 $\mathrm{mg})$ in ethyl acetate (4 $\mathrm{mL}$ ) was stirred under a hydrogen atmosphere $(1 \mathrm{~atm})$ at room temperature for $22 \mathrm{~h}$. The reaction mixture was filtered through a pad of Celite and the solvent was evaporated in vacuo. The crude product was purified by silica gel column chromatography (EtOAc/hexane 15\%,20\%, 25\%) to give the hydrogenated product 15, $(181.4 \mathrm{mg}, 76 \%)$ as an oil: $[\alpha]_{\mathrm{D}}^{26}=-24.1^{\circ}\left(\mathrm{c} 1.5, \mathrm{CHCl}_{3}\right)$; IR (neat) 3450 (br), 2968, 2936, 2860, 2361, $756 \mathrm{~cm}^{-}$ ${ }^{1}$; ${ }^{1} \mathrm{H}$ NMR $\delta\left(400 \mathrm{MHz}, \mathrm{CDCl}_{3}\right) 4.06(1 \mathrm{H}, \mathrm{dd}, J=10.0,2.8 \mathrm{~Hz}), 4.02-3.96$ (1 H, m), 3.77-3.67 (3 H, m), 3.61 $(1 \mathrm{H}, \mathrm{dd}, J=6.4,3.2 \mathrm{~Hz}), 3.58(1 \mathrm{H}, \mathrm{ddd}, J=11.2,10.8,2.8 \mathrm{~Hz}), 3.53(1 \mathrm{H}, \mathrm{dddd}, J=10.4,10.4,4.4,4.4 \mathrm{~Hz})$, $3.34(3 \mathrm{H}, \mathrm{s}), 2.02-1.95(2 \mathrm{H}, \mathrm{m}), 1.92-1.81(3 \mathrm{H}, \mathrm{m}), 1.68-1.50(3 \mathrm{H}, \mathrm{m}), 1.42-1.24(2 \mathrm{H}, \mathrm{m}), 1.20(3 \mathrm{H}, \mathrm{d}, J=$ $6.0 \mathrm{~Hz}), 1.18(1 \mathrm{H}, \mathrm{ddd}, J=12.4,10.4,10.0 \mathrm{~Hz}), 1.08(3 \mathrm{H}, \mathrm{d}, J=7.2 \mathrm{~Hz}), 1.08$ (9 H, s), 1.06 (9 H, s), $0.92(3 \mathrm{H}$, d, $J=6.8 \mathrm{~Hz}), 0.75(3 \mathrm{H}, \mathrm{d}, J=6.8 \mathrm{~Hz}) ;{ }^{13} \mathrm{C} \mathrm{NMR} \delta\left(67.8 \mathrm{MHz}, \mathrm{CDCl}_{3}\right)$ 82.9, 79.8, 73.2, 72.1, 69.3, 64.6, 55.2, 39.5, 38.8, 37.4, 36.1, 34.9, 28.7, 28.2 (3C), 27.9 (3C), 27.7, 22.3, 21.9 (2C), 15.7, 14.2, 13.2; HRMS (FD+) $[\mathrm{M}+\mathrm{H}]+$ found 473.3637, $\mathrm{C}_{26} \mathrm{H}_{53} \mathrm{O}_{5} \mathrm{Si}$ requires 473.3662 .

\section{Triol 15"}

A solution of $\mathrm{HF}$. py was prepared from $1.52 \mathrm{~g}$ of pyridinium hydrofluoride, $4.13 \mathrm{~mL}$ of pyridine, and 7.24 $\mathrm{mL}$ of THF. A mixture of alcohol 15' $(296.8 \mathrm{mg}, 0.63 \mathrm{mmol})$ and $5 \mathrm{~mL}$ of the solution of $\mathrm{HF} \cdot \mathrm{py}$ in THF (5 mL) was stirred at room temperature for $30 \mathrm{~min}$, then quenched with satd. $\mathrm{NaHCO}_{3}$. The organic layer was separated and the aqueous layer was extracted with AcOEt (x6). The combined organic layers were washed with satd. $\mathrm{CuSO}_{4}$ solution, $\mathrm{H}_{2} \mathrm{O}$ and brine, dried $\left(\mathrm{MgSO}_{4}\right)$ and concentrated in vacuo to give triol 15" which was used for 
the next step without purification: $[\alpha]_{\mathrm{D}}^{27}=-21.3^{\circ}$ (c 1.0, $\mathrm{CHCl}_{3}$ ); IR (neat) 3400 (br), 2960, 2932, 2874, 2361, 1460, 1381, $1101 \mathrm{~cm}^{-1} ;{ }^{1} \mathrm{H}$ NMR $\delta\left(270 \mathrm{MHz}, \mathrm{CDCl}_{3}\right) 4.21(1 \mathrm{H}, \mathrm{s}), 4.03(1 \mathrm{H}$, dddd, $J=4.8,4.6,4.5,4.1 \mathrm{~Hz})$, $3.90(1 \mathrm{H}, \mathrm{d}, J=9.4 \mathrm{~Hz}), 3.74(1 \mathrm{H}, \mathrm{dqd}, J=9.5,6.4,3.0 \mathrm{~Hz}), 3.70-3.64(2 \mathrm{H}, \mathrm{m}), 3.55(1 \mathrm{H}, \mathrm{tt}, J=9.6,4.6 \mathrm{~Hz})$, $3.44(1 \mathrm{H}, \mathrm{br} \mathrm{s}), 3.37-3.28(1 \mathrm{H}, \mathrm{m}), 3.34(3 \mathrm{H}, \mathrm{s}), 3.19(1 \mathrm{H}, \mathrm{d}, J=4.9 \mathrm{~Hz}), 2.02-1.92(1 \mathrm{H}, \mathrm{m}), 1.92-1.74(3 \mathrm{H}, \mathrm{m})$, $1.72(1 \mathrm{H}$, br s), $1.62(1 \mathrm{H}, \mathrm{ddd}, J=14.0,8.7,4.2 \mathrm{~Hz}), 1.43-1.18(5 \mathrm{H}, \mathrm{m}), 1.23(3 \mathrm{H}, \mathrm{d}, J=6.3 \mathrm{~Hz}), 1.07(3 \mathrm{H}, \mathrm{d}, J$ $=7.1 \mathrm{~Hz}), 0.85(3 \mathrm{H}, \mathrm{d}, J=6.8 \mathrm{~Hz}), 0.74(3 \mathrm{H}, \mathrm{d}, J=6.9 \mathrm{~Hz}) ;{ }^{13} \mathrm{C} \mathrm{NMR} \delta\left(67.8 \mathrm{MHz}, \mathrm{CDCl}_{3}\right) 80.8,77.0,73.1$, $71.8,68.8,64.8,55.2,38.3,37.1,35.6,34.7,34.5,28.9,28.8,21.6,16.2,13.0,10.9$; HRMS (FD+) [M+H]+ found 333.2626, $\mathrm{C}_{18} \mathrm{H}_{37} \mathrm{O}_{5}$ requires 333.2641.

\section{Acetal 16}

To a solution of the crude triol 15" in dry DMF $(6.3 \mathrm{~mL})$ was added p-anisaldehyde dimethyl acetal $(0.16$ $\mathrm{mL}, 0.95 \mathrm{mmol}$ ) followed by CSA $(14.6 \mathrm{mg}, 0.063 \mathrm{mmol})$ at room temperature. After stirring for $2.5 \mathrm{~h}$, the reaction mixture was diluted with a solution of hexane and $\mathrm{CH}_{2} \mathrm{Cl}_{2}(10: 1)$ and quenched with $\mathrm{H}_{2} \mathrm{O}$ and the mixture was separated. The aqueous layers were extracted with a solution of hexane and $\mathrm{CH}_{2} \mathrm{Cl}_{2}(10: 1)$ (x3). The combined organic layers were dried $\left(\mathrm{MgSO}_{4}\right)$ and concentrated in vacuo. The crude product was purified by silica gel column chromatography (EtOAc/hexane 30\%, 40\%) to give acetal $\mathbf{1 6}$ (248 $\mathrm{mg}, 87 \%$ from 15') as an oil: $[\alpha]_{\mathrm{D}}{ }^{29}=2.4^{\circ}\left(\mathrm{c} 7.4, \mathrm{CHCl}_{3}\right)$; IR (neat) 3500 (br), 2936, 2243, 1518, 1250, 910, $733 \mathrm{~cm}^{-1} ;{ }^{1} \mathrm{H}$ NMR $\delta(270$ $\left.\mathrm{MHz}, \mathrm{CDCl}_{3}\right) 7.36(2 \mathrm{H}, \mathrm{d}, J=8.6 \mathrm{~Hz}), 6.88(2 \mathrm{H}, \mathrm{d}, J=8.7 \mathrm{~Hz}), 5.48(1 \mathrm{H}, \mathrm{s}), 4.11(1 \mathrm{H}, \mathrm{dd}, J=11.2,4.6 \mathrm{~Hz})$, 4.04-3.94 (1H, m), $3.86(1 \mathrm{H}, \mathrm{dd}, J=10.1,1.7 \mathrm{~Hz}), 3.80(3 \mathrm{H}, \mathrm{s}), 3.75-3.65(1 \mathrm{H}, \mathrm{m}), 3.59-3.44$ (2H, m), 3.37$3.23(1 \mathrm{H}, \mathrm{m}), 3.33(3 \mathrm{H}, \mathrm{s}), 2.44(1 \mathrm{H}, \mathrm{d}, J=7.4 \mathrm{~Hz}), 2.19-2.07(1 \mathrm{H}, \mathrm{m}), 2.04-1.91(2 \mathrm{H}, \mathrm{m}), 1.89-1.78(2 \mathrm{H}, \mathrm{m})$, $1.73-1.51(4 \mathrm{H}, \mathrm{m}), 1.38-0.98(2 \mathrm{H}, \mathrm{m}), 1.20(3 \mathrm{H}, \mathrm{d}, J=6.3 \mathrm{~Hz}), 1.11(3 \mathrm{H}, \mathrm{d}, J=7.1 \mathrm{~Hz}), 0.92(3 \mathrm{H}, \mathrm{d}, J=6.8$ $\mathrm{Hz}), 0.76(3 \mathrm{H}, \mathrm{d}, J=6.6 \mathrm{~Hz}) ;{ }^{13} \mathrm{C} \mathrm{NMR} \delta\left(67.8 \mathrm{MHz}, \mathrm{CDCl}_{3}\right.$ ) 159.7, 130.8, 127.1 (2C), 113.4 (2C), 100.8, 82.8, 78.6, 73.1, 73.0, 71.8, 64.4, 55.0 (2C), 38.4, 35.6, 34.6, 34.4, 30.1, 29.0, 27.4, 21.6, 16.4, 11.7, 11.1; HRMS (FD+) $[\mathrm{M}]+$ found $450.2968, \mathrm{C}_{26} \mathrm{H}_{42} \mathrm{O}_{6}$ requires 450.2981 .

\section{MOM ether 16'}

To a solution of alcohol $16(248 \mathrm{mg}, 0.55 \mathrm{mmol})$ in dry $\mathrm{ClCH}_{2} \mathrm{CH}_{2} \mathrm{Cl}(3 \mathrm{~mL})$ was added ${ }^{i} \mathrm{Pr}_{2} \mathrm{NEt}(575.2 \mu \mathrm{L}$, $3.30 \mathrm{mmol})$ and $\mathrm{MOMCl}(250.8 \mu \mathrm{L}, 3.30 \mathrm{mmol})$. After being stirred for $3 \mathrm{~h}$ at $50{ }^{\circ} \mathrm{C}$, the reaction was quenched with satd. $\mathrm{NaHCO}_{3}$ solution. The mixture was separated and the aqueous layer was extracted with ether (x6). The combined organic layers were dried $\left(\mathrm{MgSO}_{4}\right)$ and concentrated in vacuo to give MOM ether 16' which was used for the next step without purification: $[\alpha]_{\mathrm{D}}^{30}=-0.64^{\circ}\left(\mathrm{c} 1.1, \mathrm{CHCl}_{3}\right.$ ); IR (neat) 2937, 2361, 1520, 1250, 1040, 912, $733 \mathrm{~cm}^{-1} ;{ }^{1} \mathrm{H}$ NMR $\delta\left(270 \mathrm{MHz}, \mathrm{CDCl}_{3}\right) 7.39(2 \mathrm{H}, \mathrm{dt}, J=9.2,2.4 \mathrm{~Hz}), 6.88(2 \mathrm{H}, \mathrm{dt}, J=9.2,2.3 \mathrm{~Hz})$, $5.42(1 \mathrm{H}, \mathrm{s}), 4.66(2 \mathrm{H}, \mathrm{s}), 4.12(1 \mathrm{H}, \mathrm{dd}, J=11.0,4.6 \mathrm{~Hz}), 4.07-3.95(1 \mathrm{H}, \mathrm{m}), 3.80(3 \mathrm{H}, \mathrm{s}), 3.75(1 \mathrm{H}, \mathrm{dd}, J=$ 10.1, $1.5 \mathrm{~Hz}), 3.74-3.63(1 \mathrm{H}, \mathrm{m}), 3.62-3.39(3 \mathrm{H}, \mathrm{m}), 3.40(3 \mathrm{H}, \mathrm{s}), 3.34(3 \mathrm{H}, \mathrm{s}), 2.17-1.77(4 \mathrm{H}, \mathrm{m}), 1.77-1.53$ 
$(3 \mathrm{H}, \mathrm{m}), 1.53-1.38(1 \mathrm{H}, \mathrm{m}), 1.34-1.12(3 \mathrm{H}, \mathrm{m}), 1.21(3 \mathrm{H}, \mathrm{d}, J=6.3 \mathrm{~Hz}), 1.01(3 \mathrm{H}, \mathrm{d}, J=6.9 \mathrm{~Hz}), 0.91(3 \mathrm{H}, \mathrm{d}, J$ $=7.1 \mathrm{~Hz}), 0.75(3 \mathrm{H}, \mathrm{d}, J=6.6 \mathrm{~Hz}) ;{ }^{13} \mathrm{C} \mathrm{NMR} \delta\left(67.8 \mathrm{MHz}, \mathrm{CDCl}_{3}\right)$ 159.7, 131.5, $127.2(2 \mathrm{C}), 113.4(2 \mathrm{C}), 100.8$, $99.1,85.7,81.6,73.3,73.2,71.4,64.5,55.9,55.1$ (2C), 38.5, 36.2, 34.8, 33.8, 30.3, 29.5, 25.3, 21.7, 17.5, 11.9, 10.0; HRMS (FD+) [M]+ found 494.3262, $\mathrm{C}_{28} \mathrm{H}_{46} \mathrm{O}_{7}$ requires 494.3244.

\section{Alcohol 16"}

To a solution of the crude acetal 16' in dry $\mathrm{CH}_{2} \mathrm{Cl}_{2}(3 \mathrm{~mL})$ was added DIBAL (1.01 $M$ solution in toluene, $1.63 \mathrm{~mL}, 1.65 \mathrm{mmol}$ ) at $0{ }^{\circ} \mathrm{C}$. After stirring for $30 \mathrm{~min}$, the reaction mixture was quenched with satd. aq. potassium sodium tartrate solution, and then the mixture was separated. The aqueous layers were extracted with AcOEt (x4). The combined organic layers were dried $\left(\mathrm{MgSO}_{4}\right)$ and concentrated in vacuo. The crude product was purified by silica gel column chromatography (EtOAc/hexane 30\%, 40\%, 50\%) to give alcohol 16" (239.6 $\mathrm{mg}, 88 \%$ from 16) as an oil: $[\alpha]_{\mathrm{D}}{ }^{30}=-1.26^{\circ}\left(\mathrm{c} 1.2, \mathrm{CHCl}_{3}\right.$ ); IR (neat) 3490 (br), 2937, 2361, 1514, 1248, 1038, $733 \mathrm{~cm}^{-1}$; ${ }^{1} \mathrm{H}$ NMR $\delta\left(270 \mathrm{MHz}, \mathrm{CDCl}_{3}\right) 7.28(2 \mathrm{H}, \mathrm{d}, J=5.6 \mathrm{~Hz}), 6.87(2 \mathrm{H}, \mathrm{d}, J=8.7 \mathrm{~Hz}), 4.67(2 \mathrm{H}, \mathrm{s}), 4.66$ $(1 \mathrm{H}, \mathrm{d}, J=10.9 \mathrm{~Hz}), 4.58(1 \mathrm{H}, \mathrm{d}, J=10.9 \mathrm{~Hz}), 4.05-3.94(1 \mathrm{H}, \mathrm{m}), 3.80(3 \mathrm{H}, \mathrm{s}), 3.78-3.59(4 \mathrm{H}, \mathrm{m}), 3.53(1 \mathrm{H}, \mathrm{tt}$, $J=10.1,4.3 \mathrm{~Hz}), 3.41(3 \mathrm{H}, \mathrm{s}), 3.34(3 \mathrm{H}, \mathrm{s}), 3.30(1 \mathrm{H}, \mathrm{dd}, J=8.6,2.6 \mathrm{~Hz}), 3.01-2.92(1 \mathrm{H}, \mathrm{m}), 2.03-1.69$ (6H, m), $1.60(1 \mathrm{H}, \mathrm{ddd}, J=13.0,10.1,5.6 \mathrm{~Hz}), 1.53-1.39(1 \mathrm{H}, \mathrm{m}), 1.35-1.14(3 \mathrm{H}, \mathrm{m}), 1.22(3 \mathrm{H}, \mathrm{d}, J=6.3 \mathrm{~Hz}), 0.97$ $(3 \mathrm{H}, \mathrm{d}, J=6.8 \mathrm{~Hz}), 0.95(3 \mathrm{H}, \mathrm{d}, J=7.1 \mathrm{~Hz}), 0.92(3 \mathrm{H}, \mathrm{d}, J=7.1 \mathrm{~Hz}) ;{ }^{13} \mathrm{C}$ NMR $\delta\left(67.8 \mathrm{MHz}, \mathrm{CDCl}_{3}\right) 158.9$, 131.0, 128.9 (2C), 113.6 (2C), 98.6, 87.7, 82.8, 82.8, 73.7, 73.1, 71.4, 64.5, 55.7, 55.1, 55.0, 39.0, 38.5, 38.4, $34.7,34.6,29.5,26.2,21.6,17.1,14.6,11.1$.

\section{Segment B (17)}

To a solution of 16" (90.8 mg, $0.183 \mathrm{mmol})$ in dry $\mathrm{CH}_{2} \mathrm{Cl}_{2}(1 \mathrm{~mL})$ was added Dess-Martin periodinane (116.3 $\mathrm{g}, 0.274 \mathrm{mmol})$. The mixture was stirred at room temperature for $30 \mathrm{~min}$ and treated with a 1:1 mixture of satd. $\mathrm{NaHCO}_{3}$ and satd. $\mathrm{Na}_{2} \mathrm{~S}_{2} \mathrm{O}_{3}$. The organic layer was separated and the aqueous layer was extracted with ether (x4). The combined organic layers were dried $\left(\mathrm{MgSO}_{4}\right)$ and concentrated in vacuo. The crude product was purified by silica gel column chromatography (EtOAc/hexane 10\%, 15\%) to give segment B (17) (83.8 mg, 93\%) as an oil: ${ }^{1} \mathrm{H}-\mathrm{NMR} \_\left(270 \mathrm{MHz}, \mathrm{CDCl}_{3}\right) 9.84(1 \mathrm{H}, \mathrm{d}, J=2.6 \mathrm{~Hz}), 7.22(2 \mathrm{H}, \mathrm{dt}, J=9.2,2.4 \mathrm{~Hz}), 6.86(2 \mathrm{H}$, $\mathrm{dt}, J=9.3,2.5 \mathrm{~Hz}), 4.69(1 \mathrm{H}, \mathrm{d}, J=6.6 \mathrm{~Hz}), 4.66(1 \mathrm{H}, \mathrm{d}, J=6.8 \mathrm{~Hz}), 4.56(1 \mathrm{H}, \mathrm{d}, J=10.9 \mathrm{~Hz}), 4.47(1 \mathrm{H}, \mathrm{d}, J$ $=10.9 \mathrm{~Hz}), 4.04(1 \mathrm{H}, \mathrm{dd}, J=7.9,1.6 \mathrm{~Hz}), 4.03-3.94(1 \mathrm{H}, \mathrm{m}), 3.80(3 \mathrm{H}, \mathrm{s}), 3.70(1 \mathrm{H}, \mathrm{tt}, J=6.9,2.8 \mathrm{~Hz}), 3.53$ $(1 \mathrm{H}, \mathrm{tt}, J=10.1,4.5 \mathrm{~Hz}), 3.41(3 \mathrm{H}, \mathrm{s}), 3.35(1 \mathrm{H}, \mathrm{dd}, J=8.6,2.6 \mathrm{~Hz}), 3.34(3 \mathrm{H}, \mathrm{s}), 2.72(1 \mathrm{H}, \mathrm{dqd}, J=7.1,7.1$, $2.6 \mathrm{~Hz}), 1.98(1 \mathrm{H}$, br d, $J=12.7 \mathrm{~Hz}), 1.89-1.68(3 \mathrm{H}, \mathrm{m}), 1.66-1.53(2 \mathrm{H}, \mathrm{m}), 1.50-1.40(1 \mathrm{H}, \mathrm{m}), 1.32-1.14(3 \mathrm{H}$, m), $1.21(3 \mathrm{H}, \mathrm{d}, J=6.3 \mathrm{~Hz}), 1.07(3 \mathrm{H}, \mathrm{d}, J=7.1 \mathrm{~Hz}), 0.97(3 \mathrm{H}, \mathrm{d}, J=6.9 \mathrm{~Hz}), 0.96(3 \mathrm{H}, \mathrm{d}, J=6.9 \mathrm{~Hz}) ;{ }^{13} \mathrm{C}$ NMR $\delta\left(67.8 \mathrm{MHz}, \mathrm{CDCl}_{3}\right)$ 204.8, 159.0, 130.8, 128.8 (2C), 113.6 (2C), 98.8, 87.2, 79.8, 73.2 (2C), 71.4, 64.7, $55.8,55.2,55.2,50.2,38.7,38.4,34.8,34.6,29.6,26.2,21.7,17.2,11.7,10.8$. 

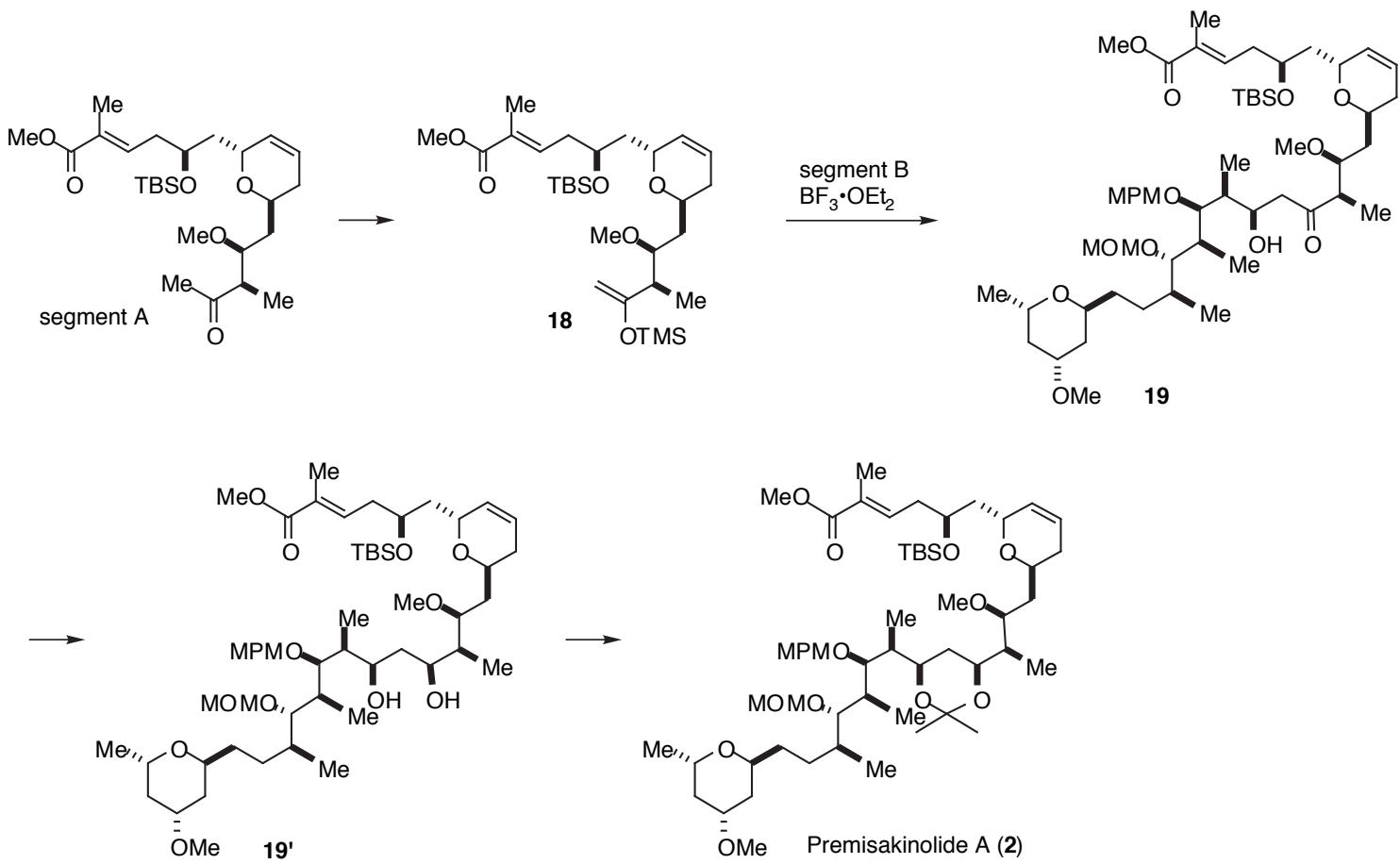

\section{Aldol compound 19}

To a solution of segment A (9) $(88.1 \mathrm{mg}, 0.183 \mathrm{mmol})$ in THF $(0.76 \mathrm{~mL})$ was added a mixture of TMSCl and $\mathrm{Et}_{3} \mathrm{~N}(1: 1$ molar ratio, $0.49 \mathrm{~mL}, 1.83 \mathrm{mmol}$ ) followed by LHMDS (a $0.62 M$ solution in THF, $0.6 \mathrm{~mL}, 0.37$ mmol) at $-78{ }^{\circ} \mathrm{C}$. After stirring for $20 \mathrm{~min}$ at $-78{ }^{\circ} \mathrm{C}$, another portion of LHMDS (1.02 $\left.\mathrm{mL}, 0.62 \mathrm{mmol}\right)$ and a mixture of $\mathrm{TMSCl}$ and $\mathrm{Et}_{3} \mathrm{~N}(0.24 \mathrm{~mL}, 0.913 \mathrm{mmol})$ were added and the resulting mixture was stirred for additional hour at the same temperature. The reaction mixture was treated with a $\mathrm{pH} 7$ buffer solution, diluted with ether, and warmed up to room temperature. The organic layer was separated and the aqueous layer was extracted with ether $(\mathrm{x} 4)$. The combined organic layers were dried $\left(\mathrm{MgSO}_{4}\right)$ and concentrated in vacuo. The residue was purified by neutral silica gel column chromatography (EtOAc/hexane 5\%) to give silyl enol ether 18 containing a small amount of impurities.

A stirred solution of the above silyl enol ether 18 and segment B (17) $(79.8 \mathrm{mg}, 0.161 \mathrm{mmol})$ in dry $\mathrm{CH}_{2} \mathrm{Cl}_{2}$ (2 mL) was cooled to $-78{ }^{\circ} \mathrm{C}$ to which $\mathrm{BF}_{3} \cdot \mathrm{OEt}_{2}(13.9 \mu \mathrm{L}, 0.11 \mathrm{mmol})$ was added dropwise. After $10 \mathrm{~min}$, another portion of $\mathrm{BF}_{3} \cdot \mathrm{OEt}_{2}(13.9 \mu \mathrm{L}, 0.11 \mathrm{mmol})$ was added and the mixture was stirred $-78{ }^{\circ} \mathrm{C}$ for an additional hour. The reaction mixture was treated with satd. $\mathrm{NaHCO}_{3}$, diluted with ether, and the aqueous layer was extracted with ether. The combined organic layers were dried $\left(\mathrm{MgSO}_{4}\right)$ and concentrated in vacuo. The oily residue was purified by neutral silica gel column chromatography (EtOAc/hexane 30\%,35\%, then 40\%) to give $19(129.9 \mathrm{mg}, 82 \%)$ as a colourless oil: $[\alpha]_{\mathrm{D}}^{30}=-28.7^{\circ}\left(\mathrm{c} 0.74, \mathrm{CHCl}_{3}\right)$; IR (neat) 3500 (br), 2936, 1711, 1514, 1250, 1088, 1038, 910, $733 \mathrm{~cm}^{-1} ;{ }^{1} \mathrm{H}$ NMR $\delta\left(270 \mathrm{MHz}, \mathrm{CDCl}_{3}\right) 7.27(2 \mathrm{H}, \mathrm{d}, J=7.1 \mathrm{~Hz}), 6.87-6.75(1 \mathrm{H}, \mathrm{m})$, 
$6.84(2 \mathrm{H}, \mathrm{d}, J=8.6 \mathrm{~Hz}), 5.77(1 \mathrm{H}, \mathrm{d}, J=10.2 \mathrm{~Hz}), 5.64(1 \mathrm{H}, \mathrm{d}, J=10.2 \mathrm{~Hz}), 4.74-4.61(4 \mathrm{H}, \mathrm{m}), 4.50(1 \mathrm{H}, \mathrm{d}, J$ $=7.3 \mathrm{~Hz}), 4.34(1 \mathrm{H}, \mathrm{d}, J=9.1 \mathrm{~Hz}), 4.16-4.04(1 \mathrm{H}, \mathrm{m}), 4.04-3.94(1 \mathrm{H}, \mathrm{m}), 3.85(1 \mathrm{H}, \mathrm{d}, J=7.4 \mathrm{~Hz}), 3.78(3 \mathrm{H}, \mathrm{s})$, 3.75-3.64 (1H, m), $3.70(3 \mathrm{H}, \mathrm{s}), 3.63-3.47(2 \mathrm{H}, \mathrm{m}), 3.41(3 \mathrm{H}, \mathrm{s}), 3.37-3.21(2 \mathrm{H}, \mathrm{m}), 3.33(3 \mathrm{H}, \mathrm{s}), 3.28(3 \mathrm{H}, \mathrm{s})$, 2.85-2.62 (2H, m), $2.53(1 \mathrm{H}, \mathrm{d}, J=17.5 \mathrm{~Hz}), 2.37(2 \mathrm{H}, \mathrm{t}, J=6.0 \mathrm{~Hz}), 2.02-1.88(4 \mathrm{H}, \mathrm{m}), 1.88-1.69(4 \mathrm{H}, \mathrm{m})$, $1.83(3 \mathrm{H}, \mathrm{s}), 1.68-1.34(7 \mathrm{H}, \mathrm{m}), 1.32-1.14(3 \mathrm{H}, \mathrm{m}), 1.21(3 \mathrm{H}, \mathrm{d}, J=6.1 \mathrm{~Hz}), 1.13-1.04(1 \mathrm{H}, \mathrm{m}), 1.09(3 \mathrm{H}, \mathrm{d}, J=$ $6.9 \mathrm{~Hz}), 0.96(3 \mathrm{H}, \mathrm{d}, J=6.8 \mathrm{~Hz}), 0.93(3 \mathrm{H}, \mathrm{d}, J=6.1 \mathrm{~Hz}), 0.89(9 \mathrm{H}, \mathrm{s}), 0.89(3 \mathrm{H}, \mathrm{d}, J=6.4 \mathrm{~Hz}), 0.12(3 \mathrm{H}, \mathrm{s})$, $0.11(3 \mathrm{H}, \mathrm{s}) ;{ }^{13} \mathrm{C} \mathrm{NMR} \delta\left(67.8 \mathrm{MHz}, \mathrm{CDCl}_{3}\right)$ 214.0, 168.3, 158.9, 138.3, 131.5, 130.2, 128.9 (2C), 128.9, 123.6, 113.6 (2C), 98.6, 87.6, 81.0, 78.6, 74.1, 73.2, 71.6, 69.1, 67.7, 66.6, 64.6, 63.9, 57.0, 55.8, 55.1 (2C), 51.6, 49.4, $46.9,41.1,40.6,38.5,38.4,37.6,36.6,34.8,34.8,30.7,29.6,26.6,25.8$ (3C), 21.7, 18.0, 17.2, 12.6, 11.2, 10.8, $10.7,-4.4,-4.7$.

\section{Diol 19'}

To a cooled solution of ketol $19(115.2 \mathrm{mg}, 0.118 \mathrm{mmol})$ in THF $(10 \mathrm{~mL})$ at $-78{ }^{\circ} \mathrm{C}$ was added catecholborane $(125.6 \mu \mathrm{L}, 1.18 \mathrm{mmol})$ and the mixture was warmed gradually to $-20{ }^{\circ} \mathrm{C}$ over $2 \mathrm{~h}$ and further stirred for $21 \mathrm{~h}$, at $-20{ }^{\circ} \mathrm{C}$. A saturated potassium sodium tartrate solution $(15 \mathrm{~mL})$ was added and stirring was continued at room temperature for $4 \mathrm{~h}$. The mixture was diluted with ether and separated. The organic layer was washed with $1 \mathrm{~N} \mathrm{NaOH}(x 2), \mathrm{H}_{2} \mathrm{O}$ and brine and the aqueous layers were extracted with ether. The combined organic layers were dried $\left(\mathrm{MgSO}_{4}\right)$ and concentrated in vacuo. The crude oil was purified by neutral silica gel column chromatography (EtOAc/hexane 30\%, 35\%, then 40\%) to give the syn-diol 19' (73.4 mg, 64\%) as a colourless oil: $[\alpha]_{\mathrm{D}}{ }^{28}=-34.7^{\circ}\left(\mathrm{c} 0.65, \mathrm{CHCl}_{3}\right.$ ); IR (neat) 3450 (br), 2936, 1713, 1514, 1462, 1250, 1086, 1038, 912, $733 \mathrm{~cm}^{-1} ;{ }^{1} \mathrm{H}$ NMR $\delta\left(270 \mathrm{MHz}, \mathrm{CDCl}_{3}\right) 7.28(2 \mathrm{H}, \mathrm{d}, J=8.6 \mathrm{~Hz}), 6.90-6.78(1 \mathrm{H}, \mathrm{m}), 6.86(2 \mathrm{H}, \mathrm{d}, J=8.7$ Hz), 5.83-5.74 (1H, m), $5.64(1 \mathrm{H}, \mathrm{d}, J=9.6 \mathrm{~Hz}), 4.69(1 \mathrm{H}, \mathrm{d}, J=6.6 \mathrm{~Hz}), 4.67(2 \mathrm{H}, \mathrm{s}), 4.65$ (1H, d, $J=6.8 \mathrm{~Hz})$, 4.39-4.23 (3H, m), 4.19-4.08 (1H, m), 4.03-3.94 (2H, m), 3.87-3.80 (1H, m), 3.79 (3H, s), 3.76-3.64 (2H, m), $3.71(3 \mathrm{H}, \mathrm{s}), 3.62-3.46(2 \mathrm{H}, \mathrm{m}), 3.42(3 \mathrm{H}, \mathrm{s}), 3.37(3 \mathrm{H}, \mathrm{s}), 3.36-3.27(2 \mathrm{H}, \mathrm{m}), 3.34(3 \mathrm{H}, \mathrm{s}), 2.41-2.32(2 \mathrm{H}, \mathrm{m})$, 2.02-1.77 (5H, m), $1.82(3 \mathrm{H}, \mathrm{s}), 1.75-1.36(11 \mathrm{H}, \mathrm{m}), 1.30-1.05(4 \mathrm{H}, \mathrm{m}), 1.21(3 \mathrm{H}, \mathrm{d}, J=6.1 \mathrm{~Hz}), 0.95(3 \mathrm{H}, \mathrm{d}, J$ $=6.9 \mathrm{~Hz}), 0.94(3 \mathrm{H}, \mathrm{d}, J=6.9 \mathrm{~Hz}), 0.89(9 \mathrm{H}, \mathrm{s}), 0.88(3 \mathrm{H}, \mathrm{d}, J=6.3 \mathrm{~Hz}), 0.83(3 \mathrm{H}, \mathrm{d}, J=7.1 \mathrm{~Hz}), 0.12(3 \mathrm{H}, \mathrm{s})$, $0.08(3 \mathrm{H}, \mathrm{s}) ;{ }^{13} \mathrm{C}$ NMR $\delta\left(67.8 \mathrm{MHz}, \mathrm{CDCl}_{3}\right)$ 168.4, 159.0, 138.5, 131.5, 130.3, 128.9 (2C), 128.8, 123.6, 113.7 (2C), 98.5, 87.6, 81.8, 79.0, 75.4, 74.2, 73.2, 71.9, 71.7, 69.1, 67.4, 64.7, 64.0, 56.9, 55.9, 55.2, 55.2, 51.6, 42.2, $40.5,40.4,39.4,38.6,38.5,37.5,36.1,34.9,34.8,31.0,29.7,26.8,25.8$ (3C), 21.7, 18.0, 17.2, 12.7, 11.5, 11.2, $10.9,-4.4,-4.7$.

\section{Protected pre-misakinolide A (2)}

To a solution of diol 19' $(72.4 \mathrm{mg}, 0.074 \mathrm{mmol})$ in dry $\mathrm{CH}_{2} \mathrm{Cl}_{2}(0.7 \mathrm{~mL})$ was added 2,2-dimethoxypropane $(54.5 \mu \mathrm{L}, 0.44 \mathrm{mmol})$ followed by CSA $(1.2 \mathrm{mg}, 5.2 \mu \mathrm{mol})$ at room temperature. After stirring for $30 \mathrm{~min}$, the 
reaction mixture was quenched with satd. $\mathrm{NaHCO}_{3}$ and the mixture was separated. The aqueous layer was extracted with ether (x4). The combined organic layers were dried $\left(\mathrm{MgSO}_{4}\right)$ and concentrated in vacuo. The crude product was purified by silica gel column chromatography (EtOAc/hexane 15\%, 20\%) to give protected pre-misakinolide A (2) $(61.8 \mathrm{mg}, 82 \%)$ as an oil: $[\alpha]_{\mathrm{D}}{ }^{28}=-38.3^{\circ}\left(\mathrm{c} 0.35, \mathrm{CHCl}_{3}\right)$; IR (neat) 2937, 1716, 1508, 1458, 1250, 1092, 1038, 910, $733 \mathrm{~cm}^{-1} ;{ }^{1} \mathrm{H}$ NMR $\delta\left(270 \mathrm{MHz}, \mathrm{CDCl}_{3}\right) 7.26(2 \mathrm{H}, \mathrm{d}, J=8.6 \mathrm{~Hz}), 6.93-6.83(1 \mathrm{H}$, m), $6.87(2 \mathrm{H}, \mathrm{d}, J=8.6 \mathrm{~Hz}), 5.83-5.73(1 \mathrm{H}, \mathrm{m}), 5.64(1 \mathrm{H}, \mathrm{d}, J=8.9 \mathrm{~Hz}), 4.68(1 \mathrm{H}, \mathrm{d}, J=6.9 \mathrm{~Hz}), 4.66(2 \mathrm{H}, \mathrm{s})$, $4.63(1 \mathrm{H}, \mathrm{d}, J=6.9 \mathrm{~Hz}), 4.38-4.23(2 \mathrm{H}, \mathrm{m}), 4.18-4.05(1 \mathrm{H}, \mathrm{m}), 4.03-3.93(1 \mathrm{H}, \mathrm{m}), 3.89-3.74(2 \mathrm{H}, \mathrm{m}), 3.80(3 \mathrm{H}$, s), 3.73-3.66 (1H, m), $3.73(3 \mathrm{H}, \mathrm{s}), 3.61-3.45(2 \mathrm{H}, \mathrm{m}), 3.42(3 \mathrm{H}, \mathrm{s}), 3.37-3.26(2 \mathrm{H}, \mathrm{m}), 3.34(3 \mathrm{H}, \mathrm{s}), 3.32(3 \mathrm{H}$, s), 2.51-2.27 (2H, m), 2.02-1.91 (2H, m), 1.91-1.76 (3H, m), $1.83(3 \mathrm{H}, \mathrm{s}), 1.76-1.51(4 \mathrm{H}, \mathrm{m}), 1.51-1.33(4 \mathrm{H}, \mathrm{m})$, $1.45(3 \mathrm{H}, \mathrm{s}), 1.38(3 \mathrm{H}, \mathrm{s}), 1.30-1.12(7 \mathrm{H}, \mathrm{m}), 1.21(3 \mathrm{H}, \mathrm{d}, J=6.1 \mathrm{~Hz}), 0.94(3 \mathrm{H}, \mathrm{d}, J=6.8 \mathrm{~Hz}), 0.89(9 \mathrm{H}, \mathrm{s})$, $0.86(3 \mathrm{H}, \mathrm{d}, J=6.8 \mathrm{~Hz}), 0.85(3 \mathrm{H}, \mathrm{d}, J=6.6 \mathrm{~Hz}), 0.79(3 \mathrm{H}, \mathrm{d}, J=6.9 \mathrm{~Hz}), 0.12(3 \mathrm{H}, \mathrm{s}), 0.09(3 \mathrm{H}, \mathrm{s}) ;{ }^{13} \mathrm{C} \mathrm{NMR}$ $\delta\left(67.8 \mathrm{MHz}, \mathrm{CDCl}_{3}\right)$ 168.4, 158.7, 138.4, 132.2, 130.3, 128.8, 128.2 (2C), 123.8, 113.6 (2C), 98.5, 98.1, 87.5, 79.0, 75.4, 73.3, 73.3, 71.6, 70.0, 69.1, 67.7, 67.5, 64.7, 64.0, 57.7, 55.7, 55.2 (2C), 51.6, 41.9, 41.2, 40.6, 38.6, 38.0, 37.4, 37.2, 34.8, 34.7, 33.0, 31.3, 30.6, 29.7, 25.9, 25.9 (3C), 21.7, 20.4, 18.0, 17.4, 12.7, 10.4, 10.2, 8.1, $4.3,-4.7$.

${ }^{1}$ (a) Nakamura, R.; Tanino, K.; Miyashita, M. Org. Lett. 2003, 5, 3579. (b) Nakamura, R.; Tanino, K.; Miyashita, M. Org. Lett. 2003, 5, 3583. 